\title{
Plants used in traditional treatment against hemorrhoids in Turkey
}

\author{
Meryem Şeyda Erbay, Aynur Sarı * \\ Department of Pharmacognosy, Faculty of Pharmacy, Istanbul University, 34116, Istanbul Turkey. \\ Correspondence: aynur@istanbul.edu.tr (A.S.); Tel: +90 2124400000 / 13581; ORCID No: 0000-0001-8116-7053.
}

Received: 31 March 2017; Revised: 23 May 2017; Accepted: 29 May 2017

\begin{abstract}
Due to its geographic location, variable climate and traditional culture, Turkey has a rich flora and the use of plants in folk medicine is very favorable. That practice and knowledge have been passed down from generation to generation. Ethnobotanical surveys are carried out to record traditional treatment methods of plants. In this study, which was prepared by screening of ethnobotanical researches made in Turkey, 241 taxa were recorded in the traditional treatment of hemorrhoids. Information about scientific and local names, families, used parts and usage patterns of these plants are given. According to the research results, the most commonly used plants in hemorrhoid treatment are plants belonging to Asteraceae, Lamiaceae, Rosaceae, Scrophulariaceae, Araceae, Polygonaceae and Cupressaceae families. The species commonly used in different regions of Turkey are Achillea sp., Arum sp., Cichorium intybus L., Dracunculus vulgaris Schott, Ecballium elaterium (L.) A. Rich., Ficus carica L., Hypericum perforatum L., H. scabrum L., Juglans regia L., Peganum harmala L., Rosa canina L., Rubus sp., Sambucus ebulus L., S. nigra L., Teucrium polium L., Urtica dioica L., Verbascum sp..
\end{abstract}

KEYWORDS: Hemorrhoids; Traditional treatment; Medicinal plants; Turkey.

\section{INTRODUCTION}

Hemorrhoids are swollen veins located around the anus or in the lower rectum. About 50 percent of adults experienced the symptoms of hemorrhoids by the age of 50. Hemorrhoids can either be internal or external. Internal hemorrhoids develop within the anus or rectum. External hemorrhoids develop outside of the anus. External hemorrhoids are the most common and most troublesome. Symptoms of hemorrhoids include irritation, pain and extreme itching around the anus, itchy or painful lump or swelling near anus, fecal leakage, painful bowel movements, blood on tissue after having a bowel movement. Possible hemorrhoids causes include straining during a bowel movement, complications from chronic constipation, sitting for a long period of time (especially on the toilet), pregnancy, obesity, a family history of hemorrhoids [1].

It is aimed to correct the underlying cause in hemorrhoid treatment. In simple cases, a fibrous diet, drinking a lot of water, local anesthetic creams for itching and pain are preferred. In symptomatic cases, treatments such as drug treatment, burning with infrared coagulant, tape-choking method, hemorrhoidal artery tying method are applied. In case progression of the disease, severe external and thrombosed hemorrhoids, definitive treatment is surgery [2].

Due to various side effects, drug interactions and high cost in synthetic drugs, interest in herbal medicines is increasing day by day. Among the population, the variety of plants used against hemorrhoids is quite high. In this study, 241 taxa were obtained against various hemorrhoids in various regions of Turkey. Scientific names, families, local names, used parts and usage of these taxa are shown in Table 1. This study includes ethnobotanical surveys made after 2004. The surveys prepared before 2004 were reported by Gürhan and Ezer [3].

How to cite this article: Erbay MŞ, Sarı A. Plants used in traditional treatment against hemorrhoids in Turkey. Marmara Pharm J. 2018; 22 (2): 110-132. 
Table 1. The plants used in traditional treatment against hemorrhoids in Turkey.

\begin{tabular}{|c|c|c|c|c|c|}
\hline Botanical name & Family & Local name & $\begin{array}{l}\text { Plant part } \\
\text { used }\end{array}$ & $\begin{array}{l}\text { Preparation, } \\
\text { administration } \\
\text { and use }\end{array}$ & Ref. \\
\hline $\begin{array}{l}\text { Achillea aleppica DC. var. } \\
\text { zederbaueri (Hayek) Hub.- } \\
\text { Mor. }\end{array}$ & Asteraceae & Yılan çiçeği & Leaf, Flower & Crushed, Ext. & [4] \\
\hline A. coarctata Poir. & Asteraceae & Mayasıl otu & Aerial part & Dried, Inf., Int. & [5] \\
\hline A. bieberstenii Afan. & Asteraceae & $\begin{array}{l}\text { Arı çiçeği, Sarı } \\
\text { papatya, } \\
\text { Erkurtaran }\end{array}$ & Aerial part & $\begin{array}{l}\text { Inf., Int. } \\
\text { Dec., Sediment Ext. }\end{array}$ & $\begin{array}{l}{[6]} \\
{[7]}\end{array}$ \\
\hline A. millefolium $\mathrm{L}$. & Asteraceae & $\begin{array}{l}\text { Civanperçemi, } \\
\text { Akbaşli, Barsam } \\
\text { otu }\end{array}$ & $\begin{array}{l}\text { Leaf, Flower } \\
\text { Aerial part }\end{array}$ & $\begin{array}{l}\text { Inf., Int. } \\
\text { Dec., Ext. }\end{array}$ & $\begin{array}{l}{[8 ; 9]} \\
{[10]}\end{array}$ \\
\hline $\begin{array}{l}\text { A. millefolium L. subsp. } \\
\text { millefolium }\end{array}$ & Asteraceae & $\begin{array}{l}\text { Beyaz } \\
\text { civanperçemi, } \\
\text { Binbir yaprak }\end{array}$ & Aerial part & $\begin{array}{l}\text { Dried, Chipped off, } \\
\text { +Honey, Eaten }\end{array}$ & {$[11 ; 12]$} \\
\hline $\begin{array}{l}\text { A. millefolium } \\
\text { L. subsp. pannonica } \\
\text { (Schelek) Hayek }\end{array}$ & Asteraceae & $\begin{array}{l}\text { Civanperçemi, } \\
\text { Dişotu, } \\
\text { Ayvadana }\end{array}$ & Aerial part & Dec., Int. & [13] \\
\hline $\begin{array}{l}\text { A. nobilis L. subsp. neilreichii } \\
\text { (Kerner) Formanek }\end{array}$ & Asteraceae & Mayasıl otu & Capitulum & Inf., Int. & (14) \\
\hline $\begin{array}{l}\text { A. nobilis L. subsp. sipylea } \\
\text { (O.Schwarz) Bassler }\end{array}$ & Asteraceae & $\begin{array}{l}\text { Kaba fesleğen, } \\
\text { Mayasıl otu }\end{array}$ & Aerial part & Inf., Int. & [14] \\
\hline A. phyrgia Boiss.\&Bal. & Asteraceae & Ayvadana & Flower & Dried, Inf., Ext. & {$[15 ; 16]$} \\
\hline A. wilhemsii C.Koch. & Asteraceae & Civanperçemi & $\begin{array}{l}\text { Leaf } \\
\text { Aerial part }\end{array}$ & $\begin{array}{l}\text { Inf., Int. } \\
\text { Dec., Ext. }\end{array}$ & $\begin{array}{l}{[17 ; 18]} \\
{[19]}\end{array}$ \\
\hline Aesculus hippocastanum L. & Hippocastanaceae & At kestanesi & Seed & Not stated & {$[15 ; 16]$} \\
\hline Aesculus sp. & Sapindaceae & At kestanesi & Seed & Crushed, Eaten & {$[20 ; 21]$} \\
\hline $\begin{array}{l}\text { Ajuga chamaepitys (L.) } \\
\text { Schreber subsp. chia } \\
\text { (Schreber) Arcangeli var. } \\
\text { chai }\end{array}$ & Lamiaceae & $\begin{array}{l}\text { Acı gicı, Mayasıl } \\
\text { otu, Kısa } \\
\text { mahmutcuk }\end{array}$ & $\begin{array}{l}\text { Aerial part } \\
\text { Flower }\end{array}$ & $\begin{array}{l}\text { Inf., Int. } \\
\text { Dec., Int. } \\
\text { Inf., Int. }\end{array}$ & $\begin{array}{l}{[22]} \\
{[19 ; 23]} \\
{[17]}\end{array}$ \\
\hline $\begin{array}{l}\text { A. chamaepitys (L.) Schreber } \\
\text { subsp. chia (Schreber) } \\
\text { Arcangeli var. ciliata Brig. }\end{array}$ & Lamiaceae & Mayasıl otu & $\begin{array}{l}\text { Flower } \\
\text { Aerial part }\end{array}$ & $\begin{array}{l}\text { Inf., Int. } \\
\text { Dec., Int. }\end{array}$ & $\begin{array}{l}{[24]} \\
{[25]}\end{array}$ \\
\hline $\begin{array}{l}\text { A. chamaepitys (L.) Schreber } \\
\text { subsp. laevigata (Banks Et } \\
\text { Sol.) P.H.Davis }\end{array}$ & Lamiaceae & Mayasıl otu & $\begin{array}{l}\text { Flower, Aerial } \\
\text { part }\end{array}$ & Dec., Int. & [21] \\
\hline Ajuga sp. & Lamiaceae & Mayasıl otu & Flower & Inf., Int. & [20] \\
\hline A. orientalis $\mathrm{L}$. & Lamiaceae & Mayasıl otu & $\begin{array}{l}\text { Whole plant } \\
\text { Flower }\end{array}$ & $\begin{array}{l}\text { Dried, Dec. } \\
\text { +Mentha longifolium } \\
\text { L. subsp. longifolium } \\
\text { (yarpuz) roots, Dec. }\end{array}$ & [26] \\
\hline $\begin{array}{l}\text { Alkanna tinctoria (L.) Tausch } \\
\text { subsp. glandulosa Hub.-Mor. }\end{array}$ & Boraginaceae & Havaciva otu & Root & Dec., Int. and Ext. & [27] \\
\hline
\end{tabular}


Table 1 (Continued). The plants used in traditional treatment against hemorrhoids in Turkey.

\begin{tabular}{|c|c|c|c|c|c|}
\hline Botanical name & Family & Local name & $\begin{array}{l}\text { Plant part } \\
\text { used }\end{array}$ & $\begin{array}{l}\text { Preparation, } \\
\text { administration } \\
\text { and use }\end{array}$ & Ref. \\
\hline Allium cepa $\mathrm{L}$. & Liliaceae & Soğan & Tuber & $\begin{array}{l}\text { Heated and } \\
\text { Crushed, Ext. }\end{array}$ & [13] \\
\hline A. porrum L. & Liliaceae & Pirasa & Seed & Inf., Int. & {$[28]$} \\
\hline A. sativum L. & Liliaceae & Sarımsak & Tuber & Swallowed & {$[29 ; 30]$} \\
\hline $\begin{array}{l}\text { Alnus glutinosa (L.) } \\
\text { Gaertn subsp. barbata } \\
\text { (C.A.Mey.) Yalt. }\end{array}$ & Betulaceae & Sakallı kızılağaç & $\begin{array}{l}\text { Seed } \\
\text { Seed, Root }\end{array}$ & $\begin{array}{l}\text { Crushed +Honey, } \\
\text { Eaten } \\
\text { Dec., Int. }\end{array}$ & {$[11 ; 12]$} \\
\hline $\begin{array}{l}\text { A. glutinosa (L.) Gaertn } \\
\text { subsp. glutinosa }\end{array}$ & Betulaceae & Kızılağaç & Male flower & Dec., Ext. & [32] \\
\hline Amaranthus retroflexus L. & Amaranthaceae & Selmik & Leaf, Branch & Inf., Int. & [17] \\
\hline Anethum graveolens L. & Apiaceae & Dere otu & $\begin{array}{l}\text { Whole plant, } \\
\text { Seed } \\
\text { Seed, Leaf }\end{array}$ & $\begin{array}{l}\text { Dec. } \\
\text { Raw, Eaten }\end{array}$ & $\begin{array}{l}{[26]} \\
{[33]}\end{array}$ \\
\hline Anthemis altissima $\mathrm{L}$. & Asteraceae & Papatya & Flower & Inf. & [9] \\
\hline A. cotula L. & Asteraceae & $\begin{array}{l}\text { Papatya, Yoğurt } \\
\text { çiçeği }\end{array}$ & Flower & $\begin{array}{l}\text { Dec., Sediment } \\
\text { Ext. }\end{array}$ & [7] \\
\hline A. nobilis L. & Asteraceae & Papatya & Flower & Inf. & [9] \\
\hline Anthemis sp. & Asteraceae & Papatya & $\begin{array}{l}\text { Flowering } \\
\text { and leafy } \\
\text { branch }\end{array}$ & Dec., Int. & [34] \\
\hline $\begin{array}{l}\text { A. tinctoria L. var. } \\
\text { tinctoria }\end{array}$ & Asteraceae & Papatya & $\begin{array}{l}\begin{array}{l}\text { Flowering } \\
\text { and leafy } \\
\text { branch }\end{array} \\
\text { Aerial part } \\
\text { Flower }\end{array}$ & $\begin{array}{l}\text { Boiled, Ext. } \\
\text { Inf. }\end{array}$ & $\begin{array}{l}{[35]} \\
{[13]} \\
{[9]}\end{array}$ \\
\hline Asphodelus aestivus Brot. & Liliaceae & $\begin{array}{l}\text { Çiriş ağusu, } \\
\text { Kiriş, Givriş, } \\
\text { Hıdırellez } \\
\text { kamçısı, Nünü }\end{array}$ & Root & $\begin{array}{l}\text { Raw, Sucked in } \\
\text { Ext. } \\
\text { Dec., Int. }\end{array}$ & {$[36 ; 37]$} \\
\hline $\begin{array}{l}\text { Asplenium adiantum- } \\
\text { nigrum L. }\end{array}$ & Aspleniaceae & Mayasıl otu & Leaf & Inf., Int. & {$[14]$} \\
\hline A. trichomanes L. & Aspleniaceae & Duvar bilonsi & Aerial part & Not stated & {$[12]$} \\
\hline $\begin{array}{l}\text { Arctium minus (Hill.) } \\
\text { Bernh. subsp. pubens } \\
\text { (Bab.) Arenes }\end{array}$ & Asteraceae & $\begin{array}{l}\text { Deve pitrağı, } \\
\text { Kaba döşeği, } \\
\text { Pitrak }\end{array}$ & Leaf & Raw, Ext. & $\begin{array}{l}{[38 ; 40 ;} \\
41]\end{array}$ \\
\hline $\begin{array}{l}\text { A. minus (Hill.) Bernh. } \\
\text { subsp. minus }\end{array}$ & Asteraceae & $\begin{array}{l}\text { Nalli pitrak, } \\
\text { Pitrak, Bitrak }\end{array}$ & Leaf & Raw, Ext. & {$[42]$} \\
\hline $\begin{array}{l}\text { Aristolochia bottae Jaub. \& } \\
\text { Spach. }\end{array}$ & Aristolochiaceae & Not stated & Aerial part & Dec., Int. & {$[43]$} \\
\hline $\begin{array}{l}\text { Arum detruncatum } \\
\text { (C.A.Meyer ex Schott.) } \\
\text { subsp. detruncatum }\end{array}$ & Araceae & $\begin{array}{l}\text { Yılan ekmeği, } \\
\text { Yılan yastığı }\end{array}$ & Tuber & $\begin{array}{l}\text { Like a pill, } \\
\text { Swallowed }\end{array}$ & [4] \\
\hline A. dioscoridis SM. & Araceae & Kabarcık, A $\breve{g}_{1}$ & Fruit & $\begin{array}{l}\text { Mature, } \\
\text { Swallowed }\end{array}$ & {$[44]$} \\
\hline $\begin{array}{l}\text { A. dioscoridis SM. var. } \\
\text { luschanii R. Mill. }\end{array}$ & Araceae & $\begin{array}{l}\text { Pancar, Yilan } \\
\text { pancarı }\end{array}$ & $\begin{array}{l}\text { Seed } \\
\text { Fruit }\end{array}$ & $\begin{array}{l}\text { Dec., Int. } \\
\text { Inf., Int. }\end{array}$ & $\begin{array}{l}{[20]} \\
{[21]}\end{array}$ \\
\hline
\end{tabular}


Table 1 (Continued). The plants used in traditional treatment against hemorrhoids in Turkey.

\begin{tabular}{|c|c|c|c|c|c|}
\hline Botanical name & Family & Local name & $\begin{array}{l}\text { Plant part } \\
\text { used }\end{array}$ & $\begin{array}{l}\text { Preparation, } \\
\text { administration } \\
\text { and use }\end{array}$ & Ref. \\
\hline $\begin{array}{l}\text { A. elongatum Steven } \\
\text { subsp. detruncatum } \\
\text { (C.A.Mey. ex Schott) H. } \\
\text { Riedl }\end{array}$ & Araceae & $\begin{array}{l}\text { Gabarcık, } \\
\text { Kabarcık }\end{array}$ & Fruit & Swallowed & {$[45 ; 46]$} \\
\hline $\begin{array}{l}\text { A. elongatum Steven } \\
\text { subsp. elongatum Steven }\end{array}$ & Araceae & $\begin{array}{l}\text { Basur otu, Yilan } \\
\text { yastığ }\end{array}$ & Tuber & $\begin{array}{l}\text { Small part with } \\
\text { bread, Swallowed }\end{array}$ & [27] \\
\hline A. italicum Miller & Araceae & $\begin{array}{l}\text { Flower otu, } \\
\text { Y1lan yastığı, } \\
\text { Yılan otu, Zehir } \\
\text { otu, Yılan } \\
\text { soğanı, Yılan } \\
\text { kılıcı, Yılancık, } \\
\text { Domuz } \\
\text { yandıran }\end{array}$ & $\begin{array}{l}\text { Fruit } \\
\text { Tuber } \\
\text { Flower }\end{array}$ & $\begin{array}{l}\text { Swallowed } \\
\text { Like a pill, } \\
\text { Swallowed } \\
\text { Inf. } \\
\text { Dec., Int. }\end{array}$ & $\begin{array}{l}\text { [32; 47; } \\
48] \\
{[49]} \\
{[32]}\end{array}$ \\
\hline A. maculatum L. & Araceae & $\begin{array}{l}\text { Tirşik, Andırın } \\
\text { doktoru, } \\
\text { Pancar, Yilan } \\
\text { ekmeği }\end{array}$ & $\begin{array}{l}\text { Tuber } \\
\text { Fruit }\end{array}$ & $\begin{array}{l}\text { Crushed, Int. } \\
\text { Dec., Int. } \\
\text { Mature, Eaten } \\
\text { Mature, Crushed, } \\
\text { +Flour, Eaten }\end{array}$ & $\begin{array}{l}{[24 ; 50]} \\
{[13]} \\
{[51]} \\
{[52]}\end{array}$ \\
\hline Artemisia absinthium L. & Asteraceae & $\begin{array}{l}\text { Ac1 yavşan, } \\
\text { Pelin otu }\end{array}$ & $\begin{array}{l}\text { Aerial part } \\
\text { Root } \\
\text { Whole plant }\end{array}$ & $\begin{array}{l}\text { +Ethanol, Mac. } \\
\text { Without Shell, } \\
\text { Swallowed } \\
\text { Dec. and Inf., Int. } \\
\text { Dec., Ext. }\end{array}$ & {$[54]$} \\
\hline Asplenium trichomanes L. & Aspleniaceae & Saçak otu & Leaf & $\begin{array}{l}\text { Achillea } \\
\text { millefolium L. and } \\
\text { Nasturtium } \\
\text { officinale } \mathrm{R.Br} \text {. } \\
\text { leaves, +Egg, } \\
\text { Cooked, Eaten }\end{array}$ & [11] \\
\hline Berberis crataegina DC. & Berberidaceae & $\begin{array}{l}\text { Zirinç, } \\
\text { Karamuk }\end{array}$ & Root & $\begin{array}{l}\text { Dec., Int. } \\
\text { Inf., Int. }\end{array}$ & $\begin{array}{l}{[19]} \\
{[55]}\end{array}$ \\
\hline $\begin{array}{l}\text { Beta corolliflora Zosimovic } \\
\text { ex Butler }\end{array}$ & Chenopodiaceae & $\begin{array}{l}\text { Kızılca, Yabani } \\
\text { 1spanak, Sırk }\end{array}$ & Root & Dec., Int. & {$[19 ; 22]$} \\
\hline $\begin{array}{l}\text { Bryonia multiflora Boiss. } \\
\text { \& Heldr. }\end{array}$ & Cucurbitaceae & $\begin{array}{l}\text { Abdulselam, } \\
\text { Akasma kökü }\end{array}$ & Root & Dec., Int. & [40] \\
\hline Caltha polypetala Hochst. & Ranunculaceae & Lilipar & Flower & Dec. & {$[26]$} \\
\hline Capparis ovata Desf. & Capparaceae & Gevil, Kafari & Root & Dec., Int. & {$[44]$} \\
\hline C. spinosa $\mathrm{L}$. & Capparaceae & Keper & Fruit & Dec., Int. & [19] \\
\hline C. spinosa L. var. spinosa & Capparaceae & $\begin{array}{l}\text { Kapari, } \\
\text { Keditırnağı }\end{array}$ & Root & Dec., Int. & {$[33]$} \\
\hline $\begin{array}{l}\text { Capsella bursa-pastoris (L.) } \\
\text { Medik }\end{array}$ & Brassicaceae & $\begin{array}{l}\text { Kuşkuş otu, } \\
\text { Çoban çantası }\end{array}$ & Leaf, Flower & Inf. & [49] \\
\hline $\begin{array}{l}\text { Cardamine raphanifolia } \\
\text { Pourr. }\end{array}$ & Brassicaceae & $\begin{array}{l}\text { Mayasıl otu, } \\
\text { Kuş lahanası }\end{array}$ & Whole plant & Eaten & {$[56]$} \\
\hline
\end{tabular}


Table 1 (Continued). The plants used in traditional treatment against hemorrhoids in Turkey.

\begin{tabular}{|c|c|c|c|c|c|}
\hline Botanical name & Family & Local name & $\begin{array}{l}\text { Plant part } \\
\text { used }\end{array}$ & $\begin{array}{l}\text { Preparation, } \\
\text { administration } \\
\text { and use }\end{array}$ & Ref. \\
\hline $\begin{array}{l}\text { Carduus acanthoides L. } \\
\text { subsp. acanthoides }\end{array}$ & Asteraceae & Küçük kenger & Aerial part & Dec., Int. & [13] \\
\hline $\begin{array}{l}\text { C. nutans L. subsp. } \\
\text { leiophyllus } \\
\text { (Petr.) Stoj. et Stef. }\end{array}$ & Asteraceae & $\begin{array}{l}\text { Deve dikeni, } \\
\text { Çakır dikeni, } \\
\text { Eşek gengeri }\end{array}$ & Aerial part & Dec., Int. & [13] \\
\hline Castanea sativa Miller & Fagaceae & Kestane & $\begin{array}{l}\text { Stem bark, } \\
\text { Fruit }\end{array}$ & Inf., Int. & [57] \\
\hline Caucalis platycarpos L. & Apiaceae & Kara pitrak & Aerial part & Dec., Int. & [58] \\
\hline Centaurea depressa Bieb. & Asteraceae & $\begin{array}{l}\text { Peygamber } \\
\text { çiçeği }\end{array}$ & Aerial part & Dec., Int. & [6] \\
\hline $\begin{array}{l}\text { C. solstitialis L. subsp. } \\
\text { solstitialis }\end{array}$ & Asteraceae & Çakır dikeni & Aerial part & Dec., Int. & [59] \\
\hline $\begin{array}{l}\text { Centaurium erythraea } \\
\text { Rafn. }\end{array}$ & Gentianaceae & $\begin{array}{l}\text { Mayasıl otu, } \\
\text { Afyonotu, } \\
\text { Kırmızı } \\
\text { kantaron }\end{array}$ & Aerial part & $\begin{array}{l}\text { Crushed, } \\
\text { +Honey, Eaten. } \\
\text { +Juglans regia L. } \\
\text { leaves and Mentha } \\
\text { sp. Dec., Ext. } \\
\text { Dried, Cooked, } \\
\text { with Gripin } \\
\text { capsule, } \\
\text { Swallowed }\end{array}$ & [47] \\
\hline $\begin{array}{l}\text { C. erythraea Roth. subsp. } \\
\text { turcicum (Velen.) }\end{array}$ & Gentianaceae & Küçük kantaron & Aerial part & Dec., Int. & {$[11 ; 12]$} \\
\hline Ceterach officinarum DC. & Aspleniaceae & Altin otu & $\begin{array}{l}\text { Leaf } \\
\text { Leaf, Flower }\end{array}$ & $\begin{array}{l}\text { Crushed and Ext. } \\
\text { Inf. }\end{array}$ & $\begin{array}{l}\text { [7] } \\
{[49]}\end{array}$ \\
\hline Chelidonium majus L. & Papaveraceae & $\begin{array}{l}\text { Kurlangıç otu, } \\
\text { Temre otu }\end{array}$ & Aerial part & Inf., Int. & [27] \\
\hline $\begin{array}{l}\text { Chenopodium album L. } \\
\text { subsp. album var. album }\end{array}$ & Chenopodiaceae & Silmik & Aerial part & Dec., Int. & [6] \\
\hline Cichorium intybus L. & Asteraceae & $\begin{array}{l}\text { Acı ot, Keklik } \\
\text { otu, Talışk, } \\
\text { Acıkök, Çıtlık, } \\
\text { Çukurca, } \\
\text { Yabani hindiba, } \\
\text { Hindiba, Sakız } \\
\text { otu, } \\
\text { Mavihindiba, } \\
\text { Radika }\end{array}$ & $\begin{array}{l}\text { Capitulum } \\
\text { Leaf } \\
\text { Leaf and stem } \\
\text { Aerial part } \\
\text { Rosette leaf } \\
\text { and root }\end{array}$ & $\begin{array}{l}\text { Dec., Int. } \\
\text { Boiled, Ext. } \\
\text { +Cucumis sativus } \\
\text { L. fruit shell, Inf., } \\
\text { Int. } \\
\text { Dec., and Cooed, } \\
\text { Int. } \\
\text { Inf., Ext. } \\
\text { Not stated }\end{array}$ & $\begin{array}{l}{[6]} \\
{[30]} \\
{[60]} \\
{[47]} \\
{[61]} \\
{[23]}\end{array}$ \\
\hline $\begin{array}{l}\text { Cirsium arvense (L.) Scop. } \\
\text { subsp. } \\
\text { vestitum (Wimm. \& } \\
\text { Grab.) Petr. }\end{array}$ & Asteraceae & Çakır dikeni & Root & Dec., Int. & [19] \\
\hline Cnicus benedictus L. & Asteraceae & $\begin{array}{l}\text { Şevketi bostan, } \\
\text { Mayasıl otu }\end{array}$ & Aerial part & Inf., Int. & [62] \\
\hline
\end{tabular}


Table 1 (Continued). The plants used in traditional treatment against hemorrhoids in Turkey.

\begin{tabular}{|c|c|c|c|c|c|}
\hline Botanical name & Family & Local name & $\begin{array}{l}\text { Plant part } \\
\text { used }\end{array}$ & $\begin{array}{l}\text { Preparation, } \\
\text { administration } \\
\text { and use }\end{array}$ & Ref. \\
\hline Convolvulus arvensis L. & Convolvulaceae & $\begin{array}{l}\text { Dağ sarmaşı̆̆ı, } \\
\text { Basırık, } \\
\text { Mahmude otu }\end{array}$ & $\begin{array}{l}\text { Root } \\
\text { Branch and } \\
\text { leaf }\end{array}$ & $\begin{array}{l}\text { Latex, Dried, } \\
\text { Swallowed } \\
\text { Dec., Ext. }\end{array}$ & $\begin{array}{l}{[63]} \\
{[7]}\end{array}$ \\
\hline Cotinus coggygria Scop. & Anacardiaceae & Tetra & Leaf & Dec., Ext. & {$[13 ; 32]$} \\
\hline Crataegus meyeri Pojark & Rosaceae & Alıç & Flower & Inf., Ext. & {$[61]$} \\
\hline $\begin{array}{l}\text { C. monogyna Jacq. subsp. } \\
\text { monogyna }\end{array}$ & Rosaceae & $\begin{array}{l}\text { Kizlarkörü, } \\
\text { Kizilçöğür, } \\
\text { Memişen }\end{array}$ & Fruit & Eaten & {$[64]$} \\
\hline $\begin{array}{l}\text { Crepis zacintha (L.) } \\
\text { Babcock }\end{array}$ & Asteraceae & Mayasıl otu & Whole plant & Dec., Int. & {$[13]$} \\
\hline Cupressus sempervirens L. & Cupressaceae & Selvi & Cone & Dec., Int. & {$[62 ; 65]$} \\
\hline $\begin{array}{l}\text { C. sempervirens L. var. } \\
\text { horizontalis }\end{array}$ & Cupressaceae & Servi & Cone & Dec. & [49] \\
\hline Cydonia oblonga Miller & Rosaceae & Ayva & Leaf & $\begin{array}{l}\text { Dried Crushed, } \\
\text { Inf., Int. }\end{array}$ & {$[32]$} \\
\hline $\begin{array}{l}\text { Cynodon dactylon (L.) } \\
\text { Pers. var. dactylon }\end{array}$ & Poaceae & $\begin{array}{l}\text { Beygir otu, } \\
\text { Ayrıkotu }\end{array}$ & $\begin{array}{l}\text { Whole plant } \\
\text { Aerial part }\end{array}$ & $\begin{array}{l}\text { Dec., Int. } \\
+ \text { Equisetum sp., } \\
\text { Dec., Int. }\end{array}$ & {$[47]$} \\
\hline Daphne sericea Vahl. & Thymelaeaceae & Zveytine, Dafne & Whole plant & $\begin{array}{l}\text { Crushed, +Olive } \\
\text { oil, Ext. }\end{array}$ & {$[20 ; 21]$} \\
\hline Datura stramonium L. & Solanaceae & $\begin{array}{l}\text { Afyonotu, Eşek } \\
\text { dikeni }\end{array}$ & Seed & Swallowed & [47] \\
\hline Daucus carota $\mathrm{L}$. & Apiaceae & $\begin{array}{l}\text { Arnamus otu, } \\
\text { Kokar otu, } \\
\text { Mayasil otu }\end{array}$ & Aerial part & Inf., Int. & {$[14]$} \\
\hline $\begin{array}{l}\text { Dianthus zonatus Fenzl. } \\
\text { var. aristatus (Boiss.) } \\
\text { Reeve }\end{array}$ & Caryophyllaceae & Basur otu & Flower & Inf., Int. & {$[27]$} \\
\hline $\begin{array}{l}\text { Dorycnium graecum (L.) } \\
\text { Ser. }\end{array}$ & Fabaceae & Dağ tifili & Flower & Dec., Int. & {$[66]$} \\
\hline $\begin{array}{l}\text { Dracunculus vulgaris } \\
\text { Schott }\end{array}$ & Araceae & $\begin{array}{l}\text { Yılan pancarı, } \\
\text { Yılan ebesi, } \\
\text { Yılan burçağı, } \\
\text { Yılan otu, Yılan } \\
\text { yastığı, Domuz } \\
\text { şekeri, } \\
\text { Kabarcık, Yılan } \\
\text { kamçısı, Yılan } \\
\text { mısırı }\end{array}$ & $\begin{array}{l}\text { Fruit } \\
\text { Root } \\
\text { Aerial part } \\
\text { Tuber and } \\
\text { seed }\end{array}$ & $\begin{array}{l}\text { Mature, +Flour, } \\
\text { Swallowed } \\
\text { Eaten } \\
\text { Like a pill, } \\
\text { Swallowed } \\
\text { +Olive oil, Dec. } \\
\text { Eaten }\end{array}$ & $\begin{array}{l}{[14 ; 42 ;} \\
62 ; 65] \\
{[33 ; 64]} \\
{[5]} \\
{[67]} \\
{[68]}\end{array}$ \\
\hline
\end{tabular}


Table 1 (Continued). The plants used in traditional treatment against hemorrhoids in Turkey.

\begin{tabular}{|c|c|c|c|c|c|}
\hline Botanical name & Family & Local name & $\begin{array}{l}\text { Plant part } \\
\text { used }\end{array}$ & $\begin{array}{l}\text { Preparation, } \\
\text { administration } \\
\text { and use }\end{array}$ & Ref. \\
\hline $\begin{array}{l}\text { Ecballium elaterium (L.) } \\
\text { A.Rich. }\end{array}$ & Cucurbitaceae & $\begin{array}{l}\text { Yaban kavunu, } \\
\text { Cirtlatan, Eşek } \\
\text { hıyarı, Acı } \\
\text { dülek, Acı } \\
\text { kavun, Deli } \\
\text { kavun, Şeytan } \\
\text { keleği, Düvelek }\end{array}$ & Fruit & $\begin{array}{l}\text { Sap, Ext. } \\
\text { Crushed, Latex } \\
\text { +Flour, Like a } \\
\text { pill, Eaten } \\
\text { Crushed, Like a } \\
\text { pill, Eaten } \\
\text { Without shell, } \\
\text { Like a pill, } \\
\text { Swallowed } \\
\text { Sliced, Ext. }\end{array}$ & $\begin{array}{l}{[20 ; 21]} \\
{[13 ; 69]} \\
\\
{[13 ; 32 ;} \\
65] \\
{[62]}\end{array}$ \\
\hline Echinops orientalis Trautv. & Asteraceae & Eşek kengeri & Flower & Dec., Int. & [6] \\
\hline Equisetum arvense $\mathrm{L}$. & Equisetaceae & $\begin{array}{l}\text { Kırk kilit otu, } \\
\text { Ulama otu, Ek } \\
\text { otu, At } \\
\text { kuyruğu, Çam } \\
\text { otu }\end{array}$ & $\begin{array}{l}\text { Stem and leaf } \\
\text { Whole plant }\end{array}$ & $\begin{array}{l}\text { Not stated } \\
\text { Dec., Ext. }\end{array}$ & $\begin{array}{l}{[8]} \\
{[54]}\end{array}$ \\
\hline Eryngium campestre L. & Apiaceae & Şeker dikeni & Root & Raw, Eaten. & [70] \\
\hline $\begin{array}{l}\text { E. campestre L. var. virens } \\
\text { Link }\end{array}$ & Apiaceae & Eşek kengeri & Root & Dec., Int. & {$[6]$} \\
\hline $\begin{array}{l}\text { Euphorbia cardiophylla } \\
\text { Boiss.et Heldr. }\end{array}$ & Euphorbiaceae & Sütleğen & Latex & Ext. & [29] \\
\hline E. kotschyana Fenzl & Euphorbiaceae & Sütleğen & Latex & Ext. & [71] \\
\hline E. macroclada Boiss. & Euphorbiaceae & Sütleğen & Latex & $\begin{array}{l}\text { Dried, Like a pill, } \\
\text { Swallowed }\end{array}$ & {$[53]$} \\
\hline E. seguieriana Necker & Euphorbiaceae & Yumru sütleğen & Latex & Ext. & [29] \\
\hline Ferula orientalis L. & Apiaceae & $\begin{array}{l}\text { Helız, Çakşır } \\
\text { otu }\end{array}$ & $\begin{array}{l}\text { Root } \\
\text { Aerial part }\end{array}$ & $\begin{array}{l}\text { Dec., Int. } \\
\text { Dec., Int. }\end{array}$ & $\begin{array}{l}{[72]} \\
{[17]}\end{array}$ \\
\hline Ferula sp. & Apiaceae & Çaşır & Root, Leaf & Dec., Int. & [73] \\
\hline Ficus carica L. & Moraceae & İncir, Yemiş & $\begin{array}{l}\text { Fruit, Leaf } \\
\text { Fruit }\end{array}$ & $\begin{array}{l}\text { Inf., Int. } \\
\text { Dec., Int. } \\
\text { Dec., Ext. } \\
\text { Raw, Ext. } \\
\text { Eaten, Compote, } \\
\text { Int. }\end{array}$ & $\begin{array}{l}{[74]} \\
{[10 ; 75]} \\
{[32]} \\
{[10]} \\
{[76]}\end{array}$ \\
\hline F. carica L. subsp. carica & Moraceae & $\begin{array}{l}\text { Deli yemiş, } \\
\text { İncir, Yemiş }\end{array}$ & $\begin{array}{l}\text { Young } \\
\text { branches }\end{array}$ & Inf., Int. & {$[64]$} \\
\hline Fragaria vesca L. & Rosaceae & Dağ çileği & Root & Dec., Ext. & {$[54]$} \\
\hline Fumaria asepala Boiss. & Papaveraceae & Şahdere & $\begin{array}{l}\text { Whole plant } \\
\text { Flower, Leaf }\end{array}$ & $\begin{array}{l}\text { Dec., Int. } \\
\text { Dec., Ext. }\end{array}$ & $\begin{array}{l}{[6]} \\
{[18]}\end{array}$ \\
\hline F. cilicica Hausskn. & Papaveraceae & Şahdere & Whole plant & Dec., Int. & [6] \\
\hline F. orientalis L. & Papaveraceae & Şahtere & Whole plant & Dec., Int. and Ext. & [26] \\
\hline
\end{tabular}


Table 1 (Continued). The plants used in traditional treatment against hemorrhoids in Turkey.

\begin{tabular}{|c|c|c|c|c|c|}
\hline Botanical name & Family & Local name & $\begin{array}{l}\text { Plant part } \\
\text { used }\end{array}$ & $\begin{array}{l}\text { Preparation, } \\
\text { administration } \\
\text { and use }\end{array}$ & Ref. \\
\hline $\begin{array}{l}\text { Galium consanguieum } \\
\text { Boiss. }\end{array}$ & Rubiaceae & Babelisk & Aerial part & Ext. & [40] \\
\hline $\begin{array}{l}\text { G. runcinatum Ehrend et } \\
\text { Schönb.-Tem. }\end{array}$ & Rubiaceae & $\begin{array}{l}\text { Yapışkan } \\
\text { sarmaşık }\end{array}$ & Aerial part & Dec., Int. & {$[6]$} \\
\hline Glycyrrhiza echinata L. & Fabaceae & Acı meyan & Root & Dec., Int. & {$[20 ; 21]$} \\
\hline $\begin{array}{l}\text { Gundelia tournefortii L. } \\
\text { var. tournefortii }\end{array}$ & Asteraceae & Kenger & Fruit & Dried, Eaten. & {$[27]$} \\
\hline $\begin{array}{l}\text { Heracleum platytaenium } \\
\text { Boiss. }\end{array}$ & Apiaceae & $\begin{array}{l}\text { Ayı göbeği, } \\
\text { Tavşan otu, Su } \\
\text { pitrağı otu }\end{array}$ & Aerial part & $\begin{array}{l}\text { +Verbascum sp. } \\
\text { aeril part, Inf., } \\
\text { Enema }\end{array}$ & {$[42 ; 77]$} \\
\hline $\begin{array}{l}\text { Hyacinthus orientalis L. } \\
\text { subsp. } \\
\text { chionophilus Wendelbo }\end{array}$ & Liliaceae & Sümbül & Leaf & Crushed, Ext. & [19] \\
\hline $\begin{array}{l}\text { Hypericum montbretii } \\
\text { Spach }\end{array}$ & Hypericaceae & $\begin{array}{l}\text { Çay çiçeği, Çay } \\
\text { otu }\end{array}$ & $\begin{array}{l}\text { Flowering } \\
\text { branch }\end{array}$ & Dec., Int. & {$[19 ; 22]$} \\
\hline H. orientale L. & Hypericaceae & $\begin{array}{l}\text { Kirmizı } \\
\text { kantaron }\end{array}$ & Aerial part & Inf., Int. & [4] \\
\hline H. perforatum $\mathrm{L}$. & Hypericaceae & $\begin{array}{l}\text { Kantaron, Sarı } \\
\text { kantaron, } \\
\text { Mayasıl otu, } \\
\text { Kizılcık otu }\end{array}$ & $\begin{array}{l}\text { Flower, Aerial } \\
\text { part }\end{array}$ & $\begin{array}{l}\text { +Olive oil, Mac., } \\
\text { Ext. } \\
\text { Crushed, +Olive } \\
\text { oil, Ext. } \\
\text { +Salix alba L. } \\
\text { leaves, Dec., Ext. } \\
\text { Dec., Int. } \\
\text { Oil, Int. }\end{array}$ & $\begin{array}{l}{[74]} \\
{[4]} \\
{[63]} \\
{[19 ; 22 ;} \\
23 ; 78] \\
{[64]}\end{array}$ \\
\hline H. scabrum L. & Hypericaceae & $\begin{array}{l}\text { Kantaron otu, } \\
\text { Sarı kantaron, } \\
\text { Serkil otu, } \\
\text { Mayasıl otu, } \\
\text { Kepir otu, } \\
\text { Kızılcık otu }\end{array}$ & $\begin{array}{l}\text { Fruit } \\
\text { Aerial part } \\
\text { Whole plant } \\
\text { Leaf, Flower }\end{array}$ & $\begin{array}{l}\text { Inf., Int. } \\
\text { Inf., Int. } \\
\text { Dec., Int. } \\
\text { Inf., Ext. } \\
\text { Dec., Int. } \\
\text { Dec. and Inf., Int., } \\
\text { Ext. }\end{array}$ & $\begin{array}{l}{[30]} \\
{[4]} \\
{[19]} \\
{[18]} \\
{[79]} \\
{[61]}\end{array}$ \\
\hline Inula anatolica Boiss. & Asteraceae & Basur otu & Flower & Dec., Ext. & {$[27]$} \\
\hline $\begin{array}{l}\text { I. helenium L. subsp. } \\
\text { vanesis Grierson }\end{array}$ & Asteraceae & $\begin{array}{l}\text { Andız otu, } \\
\text { İngüz, Peniruk }\end{array}$ & $\begin{array}{l}\text { Aerial part } \\
\text { Leaf }\end{array}$ & $\begin{array}{l}\text { Dried, Crushed, } \\
\text { +Vaseline, Ext. } \\
\text { Raw, Ext. }\end{array}$ & {$[40 ; 41]$} \\
\hline $\begin{array}{l}\text { Ixiolirion tataricum (Pall.) } \\
\text { Schult. \& Schult.f. subsp. } \\
\text { montanum (Labill.)Takht. }\end{array}$ & Amaryllidaceae & Enjurok & Fruit & Eaten & {$[80]$} \\
\hline
\end{tabular}


Table 1 (Continued). The plants used in traditional treatment against hemorrhoids in Turkey.

\begin{tabular}{|c|c|c|c|c|c|}
\hline Botanical name & Family & Local name & $\begin{array}{l}\text { Plant part } \\
\text { used }\end{array}$ & $\begin{array}{l}\text { Preparation, } \\
\text { administration } \\
\text { and use }\end{array}$ & Ref. \\
\hline Juglans regia L. & Juglandaceae & $\begin{array}{l}\text { Ceviz, Giz, Güz, } \\
\text { Koz }\end{array}$ & $\begin{array}{l}\text { Seed } \\
\text { Leaf }\end{array}$ & $\begin{array}{l}\text { Crushed, } \\
\text { +Honey, Heated, } \\
\text { Ext. } \\
\text { +Aerial part of } \\
\text { Centaurium } \\
\text { erythraea Rafn. } \\
\text { and Mentha sp., } \\
\text { Dec., Ext. } \\
\text { Dec., Int. } \\
\text { Dec., Ext. } \\
\text { Swallowed } \\
\text { Eaten } \\
\text { Mature endocarp, } \\
+ \text { Honey, Eaten. } \\
\text { Crushed, } \\
+ \text { Honey, Eaten. }\end{array}$ & $\begin{array}{l} \\
{[71 ; 81]} \\
{[19 ; 34 ;} \\
71] \\
{[60]} \\
{[13 ; 31 ;} \\
34 ; 57] \\
{[47]} \\
\\
{[14]}\end{array}$ \\
\hline Juniperus drupacea Lab. & Cupressaceae & $\begin{array}{l}\text { Andızağacı, } \\
\text { Andız }\end{array}$ & Fruit & Molasses, Int. & {$[52 ; 82]$} \\
\hline J. excelsa Bieb. & Cupressaceae & Ardiç & Fruit & Dec., Ext. & [19] \\
\hline J. oxycedrus L. & Cupressaceae & $\begin{array}{l}\text { Ardıç, Dikenli } \\
\text { ardıç, Katran } \\
\text { ardıcı }\end{array}$ & $\begin{array}{l}\text { Tar } \\
\text { Cone } \\
\text { Seed }\end{array}$ & $\begin{array}{l}\text { Like a pill, } \\
\text { Swallowed } \\
\text { and Ext. } \\
\text { Dec., Int. } \\
\text { Roasted, Crushed, } \\
\text { Int. }\end{array}$ & $\begin{array}{l}{[62 ; 65]} \\
{[15]} \\
{[32]}\end{array}$ \\
\hline $\begin{array}{l}\text { J. oxycedrus L. subsp. } \\
\text { oxycedrus }\end{array}$ & Cupressaceae & Ardıç & $\begin{array}{l}\text { Fruit } \\
\text { Cone }\end{array}$ & $\begin{array}{l}\text { Eaten } \\
\text { Inf., Int. } \\
\text { Dec., Int. }\end{array}$ & $\begin{array}{l}{[68]} \\
{[16]}\end{array}$ \\
\hline Lactuca serriola L. & Asteraceae & Hindiba, Kaju & Aerial part & Inf., Int. & [61] \\
\hline Lactuca sp. & Asteraceae & Hindiba, Kaju & Stem & Inf., Int. & [60] \\
\hline $\begin{array}{l}\text { Lauracerasus officinalis } \\
\text { Roemer }\end{array}$ & Rosaceae & $\begin{array}{l}\text { Kastanicça } \\
\text { karamişi }\end{array}$ & $\begin{array}{l}\text { Seed } \\
\text { Leaf }\end{array}$ & $\begin{array}{l}\text { Crushed, Eaten. } \\
\text { Cooked, Ext. }\end{array}$ & [56] \\
\hline Laurus nobilis L. & Lauraceae & Defne & $\begin{array}{l}\text { Oil } \\
\text { Leaf }\end{array}$ & $\begin{array}{l}\text { Ext. } \\
\text { Dec., Int. }\end{array}$ & $\begin{array}{l}{[20 ; 21]} \\
{[68]}\end{array}$ \\
\hline $\begin{array}{l}\text { Leontice leontopetalum L. } \\
\text { subsp. leontopetalum }\end{array}$ & Berberidaceae & Patlangaç & Tuber & $\begin{array}{l}\text { Sliced, Like a pill, } \\
\text { Swallowed }\end{array}$ & [69] \\
\hline $\begin{array}{l}\text { Linaria genistifolia (L.) } \\
\text { Miller subsp. genistifolia }\end{array}$ & Scrophulariaceae & Not stated & Aerial part & Dec., Int. & [6] \\
\hline
\end{tabular}


Table 1 (Continued). The plants used in traditional treatment against hemorrhoids in Turkey.

\begin{tabular}{|c|c|c|c|c|c|}
\hline Botanical name & Family & Local name & $\begin{array}{l}\text { Plant part } \\
\text { used }\end{array}$ & $\begin{array}{l}\text { Preparation, } \\
\text { administration } \\
\text { and use }\end{array}$ & Ref. \\
\hline $\begin{array}{l}\text { L. kurdica Boiss. et } \\
\text { Hohen. subsp. kurdica }\end{array}$ & Scrophulariaceae & Not stated & Aerial part & Dec., Int. & [6] \\
\hline $\begin{array}{l}\text { Liquidambar orientalis } \\
\text { Miller }\end{array}$ & Hamamelidaceae & Günlük ağacı & Kortex & Balsam & [49] \\
\hline $\begin{array}{l}\text { Lotus corniculatus L. var. } \\
\text { corniculatus (Bieb.) Arc. }\end{array}$ & Fabaceae & $\begin{array}{l}\text { Yonca, Sanc1 } \\
\text { otu, Böbrek otu }\end{array}$ & Aerial part & Dec., Int. & {$[19 ; 22]$} \\
\hline $\begin{array}{l}\text { Malabaila dasyantha (C. } \\
\text { Koch) Grossh }\end{array}$ & Apiaceae & Mandak & Leaf & $\begin{array}{l}\text { Dried or fresh, } \\
\text { Ext. }\end{array}$ & [72] \\
\hline Malva neglecta Wallr. & Malvaceae & $\begin{array}{l}\text { Ebe gömeci, Ebe } \\
\text { gümeci, Ebem } \\
\text { gömeci, Ebem } \\
\text { kömenci }\end{array}$ & $\begin{array}{l}\text { Leaf } \\
\text { Aerial part } \\
\text { Root, Seed, } \\
\text { Leaf }\end{array}$ & $\begin{array}{l}\text { Dec., Int. } \\
\text { Dec., Int. } \\
\text { Crushed, Ext. } \\
\text { +Plantago major } \\
\text { leaves, Dec. }\end{array}$ & $\begin{array}{l}{[30 ; 83]} \\
{[17 ; 18 ;} \\
70] \\
{[55]} \\
{[26]}\end{array}$ \\
\hline M. sylvestris L. & Malvaceae & Ebegümeci & Aerial part & Dec., Ext. & [13] \\
\hline $\begin{array}{l}\text { Marrubium parviflorum } \\
\text { Fisch. \& C.A. Mey. } \\
\text { subsp. parviflorum }\end{array}$ & Lamiaceae & Not stated & Whole plant & Dec., Ext. & [54] \\
\hline Matricaria chamomilla L. & Asteraceae & Papatya & Flower & Inf. & [49] \\
\hline Melia azedarach L. & Meliaceae & $\begin{array}{l}\text { Tespih ağacı, } \\
\text { Zinzra }\end{array}$ & Fruit & $\begin{array}{l}\text { Boiled, Crushed, } \\
\text { +leblebi, Mush, } \\
\text { Like a pill, } \\
\text { Swallowed }\end{array}$ & {$[20 ; 21]$} \\
\hline $\begin{array}{l}\text { Mentha longifolia L. } \\
\text { ssp. longifolia }\end{array}$ & Lamiaceae & $\begin{array}{l}\text { Yarpuz, Yarbuz, } \\
\text { Punk }\end{array}$ & $\begin{array}{l}\text { Leaf, Root } \\
\text { Aerial part }\end{array}$ & $\begin{array}{l}\text { +Camelia sinensis, } \\
\text { Inf., Ext. } \\
\text { Dec. and Inf., Int. }\end{array}$ & [19] \\
\hline Morus nigra L. & Moraceae & Karadut & Stem bark & $\begin{array}{l}\text { Peeled, Like a pill, } \\
\text { Swallowed }\end{array}$ & {$[32]$} \\
\hline Nasturtium officinale R.Br. & Brassicaceae & Su teresi & Aerial part & Raw, Eaten & {$[11 ; 12]$} \\
\hline Nepeta trachonitica Post & Lamiaceae & Dağ çayı & Aerial part & Inf., Int. & [6] \\
\hline Nerium oleander L. & Apocynaceae & Zakkum, Difli & Flower & $\begin{array}{l}\text { Crushed +butter, } \\
\text { Ext. }\end{array}$ & {$[20 ; 21]$} \\
\hline Onopordum acanthium L. & Asteraceae & Kivar & Flower & Dec., Int. & {$[31 ; 38]$} \\
\hline $\begin{array}{l}\text { O. bracteatum Boiss. et } \\
\text { Heldr. var. bracteatum } \\
\text { Boiss. et Heldr. }\end{array}$ & Asteraceae & Kangal & Seed & +Honey, Eaten & {$[19 ; 29]$} \\
\hline O. candidum Nab. & Asteraceae & Kangal & Fruit & $\begin{array}{l}\text { Crushed, Dec., } \\
\text { Int. }\end{array}$ & [6] \\
\hline $\begin{array}{l}\text { Onosma argentatum Hub.- } \\
\text { Mor. }\end{array}$ & Boraginaceae & Emzik & Aerial part & Dec., Int. & [6] \\
\hline $\begin{array}{l}\text { Orchis punctulata Steven } \\
\text { Ex Lindley }\end{array}$ & Orchidaceae & $\begin{array}{l}\text { Sahlep, Salep } \\
\text { çiçeği, Sümbül }\end{array}$ & Tuber & Dec., Int. & [6] \\
\hline Origanum onites L. & Lamiaceae & Kekik & Oil & Ext. & [84] \\
\hline
\end{tabular}


Table 1 (Continued). The plants used in traditional treatment against hemorrhoids in Turkey.

\begin{tabular}{|c|c|c|c|c|c|}
\hline Botanical name & Family & Local name & $\begin{array}{l}\text { Plant part } \\
\text { used }\end{array}$ & $\begin{array}{l}\text { Preparation, } \\
\text { administration } \\
\text { and use }\end{array}$ & Ref. \\
\hline Paliurus spina-cristi Miller & Rhamnaceae & $\begin{array}{l}\text { Arı çalısı, Kara } \\
\text { çalı, Çaltı }\end{array}$ & $\begin{array}{l}\text { Fruit } \\
\text { Seed }\end{array}$ & $\begin{array}{l}\text { Crushed, Dec., } \\
\text { Int. } \\
\text { Crushed, Inf., Int. }\end{array}$ & $\begin{array}{l}{[6 ; 44]} \\
{[24]}\end{array}$ \\
\hline Papaver rhoeas L. & Papaveraceae & Gelincik & Flower & Dec., Int. & [32] \\
\hline Peganum harmala $\mathrm{L}$. & Zygophyllaceae & $\begin{array}{l}\text { Üzerlik, } \\
\text { Harmal, Boğir, } \\
\text { Bohal meleç, } \\
\text { Yabani sedef } \\
\text { otu, İlezik, } \\
\text { Nazar otu }\end{array}$ & $\begin{array}{l}\text { Aerial part } \\
\text { Fruit } \\
\text { Seed }\end{array}$ & $\begin{array}{l}\text { Dec., Ext. } \\
\text { Burned, Cinder } \\
\text { +Barley flour and } \\
\text { olive oil, Ext. } \\
\text { Swallowed } \\
\text { Dried, Crushed } \\
\text { and Roasted, } \\
\text { Eaten } \\
\text { Crushed, } \\
+ \text { Honey, Eaten } \\
\text { Roasted, Crushed, } \\
\text { Eaten } \\
\text { Dec., Int. } \\
+ \text { Oil, Eaten } \\
\text { Chewed, Eaten } \\
\text { Crushed, Ext. } \\
\text { Dec., Int. }\end{array}$ & $\begin{array}{l}{[63]} \\
{[78]} \\
{[68 ; 85]} \\
{[78]} \\
\\
{[4 ; 65 ;} \\
71] \\
{[19 ; 23]} \\
{[62]} \\
{[76]} \\
{[57]} \\
{[19 ; 86]}\end{array}$ \\
\hline $\begin{array}{l}\text { Petroselinum arispum } \\
\text { (Mill.) A.W.Hill }\end{array}$ & Apiaceae & $\begin{array}{l}\text { Maydanoz, } \\
\text { Maydenüs }\end{array}$ & $\begin{array}{l}\text { Aerial part } \\
\text { Stem and } \\
\text { petiole }\end{array}$ & $\begin{array}{l}\text { Dec., Int. } \\
\text { Dec., Sediment } \\
\text { Ext. }\end{array}$ & $\begin{array}{l}{[87]} \\
{[7]}\end{array}$ \\
\hline Phallaris arundinaceae L. & Poaceae & Kaynaşı & Root & Dec., Int. & {$[84]$} \\
\hline $\begin{array}{l}\text { Phyllitis scolopendirum } \\
\text { (L.) Newn. }\end{array}$ & Aspleniaceae & $\begin{array}{l}\text { Geyikdili } \\
\text { eğreltisi }\end{array}$ & Aerial part & $\begin{array}{l}\text { +Viscum album L., } \\
\text { Asplenium } \\
\text { trichomanes L., } \\
\text { Plantago major L. } \\
\text { subsp. major and } \\
\text { Urtica dioica L. } \\
\text { leaves, Dec., Int. }\end{array}$ & {$[11 ; 12]$} \\
\hline Picris strigosa Bieb. & Asteraceae & Senameki & Root & Mush, Ext. & [19] \\
\hline Pinus brutia Ten. & Pinaceae & Kızılçam & Flower & $\begin{array}{l}\text { Crushed, +Water, } \\
\text { Drinked }\end{array}$ & [16] \\
\hline $\begin{array}{l}\text { Pistacia terebinthus } \\
\text { L. subsp. terebinthus }\end{array}$ & Anacardiaceae & $\begin{array}{l}\text { Kokorağaç, } \\
\text { Menengiç ağac1 }\end{array}$ & Leaf & Dec., Int. & [13] \\
\hline
\end{tabular}


Table 1 (Continued). The plants used in traditional treatment against hemorrhoids in Turkey.

\begin{tabular}{|c|c|c|c|c|c|}
\hline Botanical name & Family & Local name & $\begin{array}{l}\text { Plant part } \\
\text { used }\end{array}$ & $\begin{array}{l}\text { Preparation, } \\
\text { administration } \\
\text { and use }\end{array}$ & Ref. \\
\hline Plantago lanceolata L. & Plantaginaceae & $\begin{array}{l}\text { Giyamambel, } \\
\text { Belpanık, Sinir } \\
\text { otu, Sinirli ot, } \\
\text { Yılan dili, Yilan } \\
\text { otu }\end{array}$ & Seed & $\begin{array}{l}\text { Raw, Eaten } \\
\text { Dec., Int. } \\
\text { Dried, Crushed, } \\
\text { +Honey, Eaten } \\
\text { Crushed, Dec. }\end{array}$ & $\begin{array}{l}{[38]} \\
{[40]} \\
{[78]} \\
{[32]}\end{array}$ \\
\hline P. major L. & Plantaginaceae & $\begin{array}{l}\text { Sinir otu, Sinirli } \\
\text { ot }\end{array}$ & Leaf & Burned, Ext. & [74] \\
\hline $\begin{array}{l}\text { P. major L. subsp. } \\
\text { intermedia (Gilib.) Lange }\end{array}$ & Plantaginaceae & $\begin{array}{l}\text { Sinir otu, Sinirli } \\
\text { ot, Kesik otu, } \\
\text { Bağa yaprağı, } \\
\text { Kırkdamar otu }\end{array}$ & $\begin{array}{l}\text { Fruit } \\
\text { Leaf }\end{array}$ & $\begin{array}{l}\text { +Honey, Eaten } \\
\text { Inf., Ext. } \\
\text { Soup, Drinked } \\
\text { Grated, Crushed, } \\
\text { Ext. }\end{array}$ & $\begin{array}{l}{[5]} \\
{[19 ; 22]} \\
{[67]}\end{array}$ \\
\hline P. major L. subsp. major & Plantaginaceae & Sinir otu & $\begin{array}{l}\text { Aerial part } \\
\text { Seed } \\
\text { Leaf }\end{array}$ & $\begin{array}{l}\text { Dried, Dec. and } \\
\text { Inf., Int. } \\
\text { Dried, Eaten } \\
\text { Dec., Int. } \\
\text { Dec., Ext. }\end{array}$ & $\begin{array}{l}{[11]} \\
{[56]} \\
{[19]} \\
{[18]}\end{array}$ \\
\hline Platanus orientalis L. & Platanaceae & $\begin{array}{l}\text { Ayı otu, Eğer } \\
\text { otu, Kavak, } \\
\text { Çınar }\end{array}$ & Leaf & Inf., Int. & [64] \\
\hline Polygonum bistorta L. & Polygonaceae & Çimen eveleği & Aerial part & Dec., Int. & [11] \\
\hline P. cognatum Meisn & Polygonaceae & $\begin{array}{l}\text { Madımak, } \\
\text { Madımalak }\end{array}$ & Whole plant & Int. & [29] \\
\hline P. lapathifolium L. & Polygonaceae & $\begin{array}{l}\text { Dereotu, Dere } \\
\text { biberi, } \\
\text { Deve sürdeği }\end{array}$ & Aerial part & Dec., Ext. & [13] \\
\hline Populus tremula L. & Salicaceae & Kavak & $\begin{array}{l}\text { Gemma } \\
\text { Cortex }\end{array}$ & $\begin{array}{l}\text { +Olive oil } \\
\text { Dec., Int. }\end{array}$ & [29] \\
\hline Potentilla reptans $\mathrm{L}$. & Rosaceae & Reşadın otu & Leaf & Inf., Int. & {$[78]$} \\
\hline Prunus persica (L.) Batsch. & Rosaceae & Şeftali & Seed & Swallowed & {$[74]$} \\
\hline $\begin{array}{l}\text { Pteridum aquilinum (L.) } \\
\text { Kuhn }\end{array}$ & Dennstaedtiaceae & Kartal eğrisi & Leaf & Not stated & [88] \\
\hline Pteris cretica L. & Pteridaceae & Girit eğreltisi & Root & $\begin{array}{l}\text { +Phedimus } \\
\text { stoloniferus } \\
\text { (S.G.Gmel.), } \\
\text { Urtica dioica L., } \\
\text { Hedera helix L., } \\
\text { Ficus L., } \\
\text { Laurocerasus } \\
\text { Duhamel, Juglans } \\
\text { and Alnus leaves, } \\
\text { Dec., Int. }\end{array}$ & {$[11]$} \\
\hline Punica granatum L. & Punicaceae & Nar, Mekke gülü & Fruit bark & Dec., Int. & [8] \\
\hline
\end{tabular}


Table 1 (Continued). The plants used in traditional treatment against hemorrhoids in Turkey.

\begin{tabular}{|c|c|c|c|c|c|}
\hline Botanical name & Family & Local name & $\begin{array}{l}\text { Plant part } \\
\text { used }\end{array}$ & $\begin{array}{l}\text { Preparation, } \\
\text { administration } \\
\text { and use }\end{array}$ & Ref. \\
\hline $\begin{array}{l}\text { Quercus cerris L. var. } \\
\text { cerris }\end{array}$ & Fagaceae & Kızılmeşe, Meşe & Fruit & Dec., Int. & [27] \\
\hline Q. coccifera $\mathrm{L}$. & Fagaceae & Meşe & $\begin{array}{l}\text { Fruit, Gall, } \\
\text { Leaf }\end{array}$ & Not stated & {$[15 ; 16]$} \\
\hline Q. libani Oliver & Fagaceae & Meşe & Fruit & Eaten & [6] \\
\hline $\begin{array}{l}\text { Q. petraea (Mattuschka) } \\
\text { Liebl. subsp. pinnatiloba } \\
\text { (C.Koch) Menitsky }\end{array}$ & Fagaceae & Meşe & Fruit & Eaten & [6] \\
\hline Ranunculus ficaria L. & Ranunculaceae & Basur otu & Root & Boiled, Mush, Ext. & [89] \\
\hline $\begin{array}{l}\text { R. ficaria L. subsp. } \\
\text { ficariiformis Rouy et Fouc. }\end{array}$ & Ranunculaceae & $\begin{array}{l}\text { Altın tabak, } \\
\text { Düğün çiçeği, } \\
\text { Basur otu, Sarı } \\
\text { çiçek, Mayıs } \\
\text { çiçeği }\end{array}$ & $\begin{array}{l}\text { Rosette leaf } \\
\text { Flower } \\
\text { Root } \\
\text { Aerial part }\end{array}$ & $\begin{array}{l}\text { Mush Ext. } \\
\text { Inf., Int. } \\
\text { Crushed, Eaten } \\
\text { Crushed, Ext. }\end{array}$ & $\begin{array}{l}{[20]} \\
{[27]} \\
{[69]} \\
{[21]}\end{array}$ \\
\hline Reseda lutea L. & Resedaceae & Eşek gerdanası & Aerial part & Inf., Int. & {$[4]$} \\
\hline Rheum ribes $\mathrm{L}$. & Polygonaceae & $\begin{array}{l}\text { Işgın, Işkın, } \\
\text { Rives, Gavalak, } \\
\text { Gavalat }\end{array}$ & Root & $\begin{array}{l}\text { Dec., Int. } \\
\text { Roasted, +Honey, } \\
\text { Swallowed }\end{array}$ & $\begin{array}{l}{[6 ; 19 ; 22 ;} \\
86 ; 90] \\
{[91]}\end{array}$ \\
\hline Rosa canina L. & Rosaceae & $\begin{array}{l}\text { Kuşburnu, } \\
\text { Şillan, Şilan, } \\
\text { Yabani gül, Gül } \\
\text { elması, } \\
\text { Gülburnu, } \\
\text { İtburnu }\end{array}$ & $\begin{array}{l}\text { Flower, Fruit, } \\
\text { Root } \\
\text { Fruit }\end{array}$ & $\begin{array}{l}\text { Not stated } \\
\text { Dec., Int. } \\
\text { Dried, } \\
\text { Marmalade, Int. } \\
\text { Raw, Eaten } \\
\text { Dried, Eaten } \\
\text { Inf., Int. and Ext. } \\
\text { Dec., Int. } \\
\text { Dec., Int. } \\
\text { Dried cortex, } \\
\text { Crushed and } \\
\text { Dec., Int. }\end{array}$ & $\begin{array}{l}{[89]} \\
{[6 ; 19 ; 30 ;} \\
64 ; 78] \\
{[27 ; 63]} \\
{[68]} \\
{[55]} \\
{[31]} \\
{[20 ; 21]} \\
{[19 ; 32]} \\
{[72]}\end{array}$ \\
\hline $\begin{array}{l}\text { R. dumalis Bechst. var. } \\
\text { boissieri (Chepin) O. } \\
\text { Nilsson }\end{array}$ & Rosaceae & Kuşburnu & Fruit & $\begin{array}{l}\text { With seed, Eaten } \\
\text { and Mush }\end{array}$ & [91] \\
\hline $\begin{array}{l}\text { R. montana Chaix subsp. } \\
\text { woronowii (Lonacz.) } \\
\text { Ö.Nilsson }\end{array}$ & Rosaceae & Kuşburnu & Fruit, Flower & $\begin{array}{l}\text { Marmalade, juice } \\
\text { and tea, Int. }\end{array}$ & [29] \\
\hline
\end{tabular}


Table 1 (Continued). The plants used in traditional treatment against hemorrhoids in Turkey.

\begin{tabular}{|c|c|c|c|c|c|}
\hline Botanical name & Family & Local name & $\begin{array}{l}\text { Plant part } \\
\text { used }\end{array}$ & $\begin{array}{l}\text { Preparation, } \\
\text { administration } \\
\text { and use }\end{array}$ & Ref. \\
\hline R. pimpinellifolia L. & Rosaceae & $\begin{array}{l}\text { Koyungözü, } \\
\text { Atgötü }\end{array}$ & Fruit & Dec., Int. & {$[19 ; 22]$} \\
\hline $\begin{array}{l}\text { R. villosa L. subsp. mollis } \\
\text { (SM.) Keller et Gams }\end{array}$ & Rosaceae & Kıllı kuşburnu & Fruit, Flower & $\begin{array}{l}\text { Marmalade, juice } \\
\text { and tea, Int. }\end{array}$ & [29] \\
\hline Rubus caesius L. & Rosaceae & $\begin{array}{l}\text { Böğürtlen, } \\
\text { Mora, Fuska } \\
\text { dikeni, Pamuk } \\
\text { dikeni, } \\
\text { Handuka, } \\
\text { Fiskofi }\end{array}$ & $\begin{array}{l}\text { Fruit } \\
\text { Root }\end{array}$ & $\begin{array}{l}\text { Marmalade, } \\
\text { Compote, Int. } \\
\text { Cooked, Eaten }\end{array}$ & $\begin{array}{l}{[29]} \\
{[56]}\end{array}$ \\
\hline $\begin{array}{l}\text { R. canescens DC. var. } \\
\text { canescens }\end{array}$ & Rosaceae & Böğürtlen & Leaf and Fruit & Dec., Int. & [23] \\
\hline $\begin{array}{l}\text { R. canescens var. glabratus } \\
\text { (Godron) Davis et Meikle }\end{array}$ & Rosaceae & $\begin{array}{l}\text { Böğürtlen, } \\
\text { Mora }\end{array}$ & Fruit & $\begin{array}{l}\text { Marmalade, } \\
\text { Compote, Int. }\end{array}$ & [29] \\
\hline $\begin{array}{l}\text { R. discolor Wheihe \& } \\
\text { Nees. }\end{array}$ & Rosaceae & Böğürtlen & Root & Dec., Int. & [28] \\
\hline R. sanctus Schreber & Rosaceae & Böğürtlen & $\begin{array}{l}\text { Leaf and } \\
\text { Flower } \\
\text { Root }\end{array}$ & $\begin{array}{l}\text { Crushed, Ext. } \\
\text { Dec., Int. }\end{array}$ & $\begin{array}{l}{[20 ; 21]} \\
{[92]}\end{array}$ \\
\hline R. saxatilis L. & Rosaceae & $\begin{array}{l}\text { Böğürtlen, } \\
\text { Mora }\end{array}$ & Fruit & $\begin{array}{l}\text { Marmalade, } \\
\text { Compote, Int. }\end{array}$ & [29] \\
\hline Rumex crispus L. & Polygonaceae & $\begin{array}{l}\text { Adem eveliği, } \\
\text { Evelik }\end{array}$ & Leaf & $\begin{array}{l}\text { Inf., Int. } \\
\text { Crushed, Ext. }\end{array}$ & $\begin{array}{l}{[19 ; 22]} \\
{[86]}\end{array}$ \\
\hline $\begin{array}{l}\text { R. obtusifolius L. subsp. } \\
\text { subalpinus } \\
\text { (Schur) Celak. }\end{array}$ & Polygonaceae & Lapaza & Seed & Dried, Dec., Int. & {$[56]$} \\
\hline R. patientia $\mathrm{L}$. & Polygonaceae & Kuzukulağı & $\begin{array}{l}\text { Fruit } \\
\text { Leaf }\end{array}$ & $\begin{array}{l}\text { Dec., Int. } \\
\text { Inf., Int. }\end{array}$ & $\begin{array}{l}{[6]} \\
{[19]}\end{array}$ \\
\hline Rumex sp. & Polygonaceae & Labada, İlibada & Seed & Dried, Dec., Ext. & [74] \\
\hline Salix alba $\mathrm{L}$. & Salicaceae & Söğüt & Leaf & $\begin{array}{l}+ \text { Hypericum } \\
\text { perforatum L., } \\
\text { Dec., Ext. }\end{array}$ & [63] \\
\hline $\begin{array}{l}\text { Salvia cryptantha } \\
\text { Montbret et Aucher ex } \\
\text { Bentham }\end{array}$ & Lamiaceae & $\begin{array}{l}\text { Adaçayı, Çalba, } \\
\text { Kayışkıran, } \\
\text { Kokulu ot, Sarı } \\
\text { şabla }\end{array}$ & Aerial part & Inf., Int. & {$[30 ; 83]$} \\
\hline Sambucus ebulus L. & Caprifoliaceae & $\begin{array}{l}\text { Mürver, Otsu } \\
\text { mürver, Cüce } \\
\text { mürver, Ayı } \\
\text { boğan, } \\
\text { Şahmelek otu, } \\
\text { Sultan, Piran, } \\
\text { Lüver, Lor, } \\
\text { Memer }\end{array}$ & $\begin{array}{l}\text { Seed } \\
\text { Aerial part }\end{array}$ & $\begin{array}{l}\text { Mature, } \\
\text { Swallowed } \\
\text { Eaten } \\
\text { Dec., Int. } \\
\text { Eaten } \\
\text { Dec., Ext. }\end{array}$ & $\begin{array}{l}{[11 ; 32 ;} \\
93 ; 94] \\
{[47 ; 56]} \\
{[13 ; 32]} \\
{[24]} \\
{[32]}\end{array}$ \\
\hline
\end{tabular}


Table 1 (Continued). The plants used in traditional treatment against hemorrhoids in Turkey.

\begin{tabular}{|c|c|c|c|c|c|}
\hline Botanical name & Family & Local name & $\begin{array}{l}\text { Plant part } \\
\text { used }\end{array}$ & $\begin{array}{l}\text { Preparation, } \\
\text { administration } \\
\text { and use }\end{array}$ & Ref. \\
\hline S. nigra L. & Caprifoliaceae & $\begin{array}{l}\text { Patpatik, } \\
\text { Mürver, Siyah } \\
\text { mürver, Köpek } \\
\text { üzümü, Lüver, } \\
\text { Lor, Sultan, } \\
\text { Piran, Piren, } \\
\text { Sultanotu, } \\
\text { Melikşah }\end{array}$ & $\begin{array}{l}\text { Flower } \\
\text { Fruit } \\
\text { Seed }\end{array}$ & $\begin{array}{l}\text { Inf., Int. } \\
\text { Dec., Int. } \\
\text { Mature, } \\
\text { Swallowed } \\
\text { Crushed, Ext. } \\
\text { Dec., Enema } \\
\text { Inf., Int. }\end{array}$ & $\begin{array}{l}{[6]} \\
{[13]} \\
{[11 ; 47 ;} \\
63] \\
{[57]} \\
{[42 ; 77]} \\
{[13]}\end{array}$ \\
\hline Scolymus hispanicus L. & Asteraceae & Suluca diken & Root & $\begin{array}{l}\text { Without shell, } \\
\text { Crushed, Eaten }\end{array}$ & [58] \\
\hline $\begin{array}{l}\text { Scutelleria orientalis L. } \\
\text { subsp. pichleri (Stapf.) } \\
\text { Edmondson }\end{array}$ & Lamiaceae & Keselmahmut & Aerial part & $\begin{array}{l}\text { Dried, Crushed, } \\
\text { Dec., Int. }\end{array}$ & {$[38]$} \\
\hline $\begin{array}{l}\text { S. orientalis L. subsp. } \\
\text { virens (Boiss. \& Kotschy.) } \\
\text { Edmondson }\end{array}$ & Lamiaceae & Keselmahmut & Aerial part & $\begin{array}{l}\text { Dried, Crushed, } \\
\text { Dec., Int. }\end{array}$ & {$[38]$} \\
\hline $\begin{array}{l}\text { Sideritis bilgeriana } \\
\text { P.H.Davis }\end{array}$ & Lamiaceae & $\begin{array}{l}\text { Boz şabla, } \\
\text { Kekik çayı, } \\
\text { Yayla çayı }\end{array}$ & Aerial part & Inf., Ext. & {$[4]$} \\
\hline Silene saxatilis Sims & Caryophyllaceae & $\begin{array}{l}\text { Mayasıl otu, } \\
\text { Zembil, Gelin } \\
\text { parmağı, Has } \\
\text { pancarı }\end{array}$ & Leaf & Inf., Int. & {$[90]$} \\
\hline $\begin{array}{l}\text { S. vulgaris (Moench) } \\
\text { Garcke }\end{array}$ & Caryophyllaceae & $\begin{array}{l}\text { Mayasıl otu, } \\
\text { Zembil, Gelin } \\
\text { parmağı }\end{array}$ & Leaf & Inf., Int. & [90] \\
\hline Silybum marianum L. & Asteraceae & Deve dikeni & Flower & $\begin{array}{l}\text { Dried, Smoked } \\
\text { Dec., Int. }\end{array}$ & {$[44]$} \\
\hline Solanum melongena L. & Solanaceae & Patlican & $\begin{array}{l}\text { Fruit stalk } \\
\text { Root }\end{array}$ & $\begin{array}{l}\text { Dec., Int. } \\
\text { Burned, Embers, } \\
\text { Ext. }\end{array}$ & $\begin{array}{l}{[74]} \\
{[52]}\end{array}$ \\
\hline S. nigrum L. & Solanaceae & İt üzümü & $\begin{array}{l}\text { Leaf, Flower } \\
\text { and Sprout }\end{array}$ & Inf. & [49] \\
\hline Sorbus aucuparia L. & Rosaceae & Üvez & Fruit & Raw, Eaten & [13] \\
\hline Stachys arvensis L. & Lamiaceae & $\begin{array}{l}\text { Karabaş, Koca } \\
\text { soğulcan }\end{array}$ & Aerial part & Dried, Dec., Int. & {$[11 ; 12]$} \\
\hline $\begin{array}{l}\text { Telephium imperati L. } \\
\text { subsp. orientale } \\
\text { (Boiss.) Nyman }\end{array}$ & Caryophyllaceae & Mayasıl otu & Leaf & Dec., Ext. & {$[19 ; 86]$} \\
\hline Terminalia chebula Retz. & Combretaceae & Karahelile & Seed & Not stated & {$[89 ; 95]$} \\
\hline Teucrium chamaedrys L. & Lamiaceae & $\begin{array}{l}\text { Kisa mahmut, } \\
\text { Mayasıl otu, } \\
\text { Sanc1 otu }\end{array}$ & Aerial part & Inf., Int. & {$[4]$} \\
\hline
\end{tabular}


Table 1 (Continued). The plants used in traditional treatment against hemorrhoids in Turkey.

\begin{tabular}{|c|c|c|c|c|c|}
\hline Botanical name & Family & Local name & $\begin{array}{l}\text { Plant part } \\
\text { used }\end{array}$ & $\begin{array}{l}\text { Preparation, } \\
\text { administration } \\
\text { and use }\end{array}$ & Ref. \\
\hline $\begin{array}{l}\text { T. chamaedrys L. subsp. } \\
\text { chamaedrys }\end{array}$ & Lamiaceae & $\begin{array}{l}\text { Bodurmahmut, } \\
\text { Sancı otu, } \\
\text { Çoban sargısı, } \\
\text { Kisacık } \\
\text { mahmut, } \\
\text { Mayasıl otu }\end{array}$ & $\begin{array}{l}\text { Leaf, Flower } \\
\text { Aerial part }\end{array}$ & $\begin{array}{l}\text { Inf., Int. } \\
\text { Crushed, Inf., and } \\
\text { Dec., Int. } \\
\text { Dec., Int. and Ext. }\end{array}$ & $\begin{array}{l}{[27]} \\
{[32 ; 53]} \\
{[78]}\end{array}$ \\
\hline $\begin{array}{l}\text { T. chamaedrys L. subsp. } \\
\text { sinuatum (Celak.) Rech. } \\
\text { Fil. }\end{array}$ & Lamiaceae & Dağ kekiği & Aerial part & Inf., Int. & [6] \\
\hline $\begin{array}{l}\text { T. chamaedrys L. subsp. } \\
\text { tauricolum Rech. Fil. }\end{array}$ & Lamiaceae & Dağ kekiği & Aerial part & Inf., Int. & [24] \\
\hline T. parviflorum Schreber & Lamiaceae & Dağ kekiği & Aerial part & Dec., Int. & {$[6 ; 19]$} \\
\hline T. polium L. & Lamiaceae & $\begin{array}{l}\text { Yavşan, Mayasıl } \\
\text { otu, Acı ot, Ak } \\
\text { sedef otu, Basur } \\
\text { otu, Meryem } \\
\text { otu, Sancı otu, } \\
\text { Koyun otu, } \\
\text { Bozot, Acı } \\
\text { yavşan, Oğlan } \\
\text { otu, Oğul otu, } \\
\text { Peryavşan, } \\
\text { Tiksinik otu }\end{array}$ & $\begin{array}{l}\text { Leaf, Branch } \\
\text { and Flower } \\
\text { Stem } \\
\text { Aerial part }\end{array}$ & $\begin{array}{l}\text { Inf., Int. } \\
\text { Dec., Int. and Ext. } \\
\text { Dried, Dec., Int. } \\
\text { Dried, Crushed, } \\
\text { +Vitis vinifera L. } \\
\text { fruits, Eaten } \\
\text { Dec., Ext. } \\
\text { Inf., Int. } \\
\text { Crushed, } \\
+ \text { Honey, Eaten }\end{array}$ & $\begin{array}{l}\text { [79; 82; } \\
89] \\
{[27]} \\
\\
{[19 ; 71 ;} \\
78 ; 96] \\
{[96]} \\
\\
{[44 ; 78]} \\
{[14 ; 55 ;} \\
64] \\
{[64]}\end{array}$ \\
\hline Thuja arborvitae L. & Cupressaceae & Mazı & Leaf & Not stated & [89] \\
\hline $\begin{array}{l}\text { Thymus fallax Fisch. \& } \\
\text { C.A. Mey. }\end{array}$ & Lamiaceae & Catri & $\begin{array}{l}\text { Leafy young } \\
\text { branch } \\
\text { Leaf }\end{array}$ & $\begin{array}{l}\text { Dec., Int. } \\
\text { Raw, Eaten,Ext. }\end{array}$ & {$[72]$} \\
\hline $\begin{array}{l}\text { T. leucostomus Hausskn. } \\
\text { \& Velen. var. leucostomus }\end{array}$ & Lamiaceae & $\begin{array}{l}\text { Paryavşan, } \\
\text { Kekik }\end{array}$ & Aerial part & Inf., Int. & {$[70]$} \\
\hline Thymus sp. & Lamiaceae & $\begin{array}{l}\text { Kekik, Kesik } \\
\text { otu }\end{array}$ & Aerial part & Dec., Int. & [28] \\
\hline Tribulus terrestis L. & Zygophyllaceae & $\begin{array}{l}\text { Deve çökerten, } \\
\text { Demir dikeni, } \\
\text { Deve dikeni, } \\
\text { Çoban çökerten }\end{array}$ & $\begin{array}{l}\text { Aerial part } \\
\text { Oil } \\
\text { Leaf and Fruit }\end{array}$ & $\begin{array}{l}\text { Dec., Int. } \\
\text { Dec. and Inf., Int. } \\
\text { Ext. } \\
\text { Inf., Int. }\end{array}$ & $\begin{array}{l}{[8]} \\
{[33]} \\
{[27]} \\
{[49 ; 61]}\end{array}$ \\
\hline Trifolium ambiguum Bieb. & Fabaceae & $\begin{array}{l}\text { Alma otu, Eşek } \\
\text { yoncası, Üç } \\
\text { kulak otu }\end{array}$ & Aerial part & Dec., Int. & {$[22 ; 19]$} \\
\hline $\begin{array}{l}\text { Trigonella foenum-graecum } \\
\text { L. }\end{array}$ & Fabaceae & Çemen & Seed & Oil & [76] \\
\hline
\end{tabular}


Table 1 (Continued). The plants used in traditional treatment against hemorrhoids in Turkey.

\begin{tabular}{|c|c|c|c|c|c|}
\hline Botanical name & Family & Local name & $\begin{array}{l}\text { Plant part } \\
\text { used }\end{array}$ & $\begin{array}{l}\text { Preparation, } \\
\text { administration } \\
\text { and use }\end{array}$ & Ref. \\
\hline $\begin{array}{l}\text { Ulmus minor Miller } \\
\text { subsp. canescens } \\
\text { (Melville) Browicz et } \\
\text { Zielinski }\end{array}$ & Ulmaceae & Kara ağaç & Root & $\begin{array}{l}\text { Shell, Dec., } \\
\text { +Wheat, Mush, } \\
\text { Ext. }\end{array}$ & [30] \\
\hline Urtica dioica $\mathrm{L}$. & Urticaceae & $\begin{array}{l}\text { Isırgan otu, } \\
\text { Kırros, Isırgan, } \\
\text { Acı ısırgan, } \\
\text { Büyük 1sırgan } \\
\text { otu, Sırgan, } \\
\text { Erkek çakır, } \\
\text { Eşek çakırı, } \\
\text { Eşek 1sırganı, } \\
\text { Dalagan, } \\
\text { Gizirkan, Deli } \\
\text { ısırgan }\end{array}$ & $\begin{array}{l}\text { Seed } \\
\text { Leaf } \\
\text { Whole plant }\end{array}$ & $\begin{array}{l}\text { Dec., Int. } \\
\text { Dried, Inf., Int. } \\
\text { and Ext., Enema } \\
\text { Inf., Int. } \\
\text { +Mentha longifolia } \\
\text { (L.) Huds., Dec., } \\
\text { Int. and Ext. } \\
\text { +Honey, Eaten } \\
+ \text { +Phyllitis } \\
\text { scolopendrium (L.) } \\
\text { Newn., Dec., } \\
\text { +Honey, Int. } \\
\text { Dec., Int. } \\
\text { Dec. and Inf., Int. }\end{array}$ & $\begin{array}{l}{[13 ; 20 ;} \\
21 ; 28] \\
{[42]} \\
\\
{[14 ; 31 ;} \\
42] \\
{[78]}\end{array}$ \\
\hline Urtica sp. & Urticaceae & Isırgan & Seed & Inf., Int. & {$[60]$} \\
\hline $\begin{array}{l}\text { Verbascum } \\
\text { cheiranthifolium Boiss. } \\
\text { var. cheiranthifolium }\end{array}$ & Scrophulariaceae & $\begin{array}{l}\text { Balık otu, } \\
\text { Sığırkuyruğu }\end{array}$ & $\begin{array}{l}\text { Aerial part } \\
\text { Flower and } \\
\text { Sprout } \\
\text { Root and Base } \\
\text { leaf }\end{array}$ & $\begin{array}{l}\text { Dec., Int. } \\
\text { Ext. } \\
\text { Dec., Int. } \\
\text { Dried, Dec., Int. } \\
\text { Dried, Crushed, } \\
\text { Dec., Int. }\end{array}$ & $\begin{array}{l}{[6]} \\
{[38]} \\
{[71 ; 81]} \\
{[96]}\end{array}$ \\
\hline $\begin{array}{l}\text { V. dudleyanum (Hub.- } \\
\text { Mor.) Hub.- Mor. }\end{array}$ & Scrophulariaceae & Sığırkuyruğu & Flower, Leaf & +Sugar, Dec. & [26] \\
\hline V. glomeratum Boiss. & Scrophulariaceae & Sığırkuyruğu & $\begin{array}{l}\text { Leaf and } \\
\text { Flower }\end{array}$ & Inf. and Dec., Int. & [53] \\
\hline $\begin{array}{l}\text { V. lasianthum Boiss. et } \\
\text { Bentham }\end{array}$ & Scrophulariaceae & $\begin{array}{l}\text { Sı̆̆ırkuyruğu, } \\
\text { Yalangı, Yılang1 }\end{array}$ & $\begin{array}{l}\text { Flower } \\
\text { Root }\end{array}$ & $\begin{array}{l}\text { Inf., Int. } \\
\text { Dried, Crushed } \\
\text { +Raisins, Eaten }\end{array}$ & $\begin{array}{l}{[30 ; 83]} \\
{[43]}\end{array}$ \\
\hline $\begin{array}{l}\text { V. oreophilum C.Koch var. } \\
\text { joannis (Bordz.) Hub.- } \\
\text { Mor. }\end{array}$ & Scrophulariaceae & Masicerk & Aerial part & Dec., Int. & {$[38]$} \\
\hline V. pyramidatum M. Bieb. & Scrophulariaceae & Masicerk & Aerial part & Dec., Int. & [38] \\
\hline
\end{tabular}


Table 1 (Continued). The plants used in traditional treatment against hemorrhoids in Turkey.

\begin{tabular}{|c|c|c|c|c|c|}
\hline Botanical name & Family & Local name & $\begin{array}{l}\text { Plant part } \\
\text { used }\end{array}$ & $\begin{array}{l}\text { Preparation, } \\
\text { administration } \\
\text { and use }\end{array}$ & Ref. \\
\hline $\begin{array}{l}\text { V. sinuatum L. var. } \\
\text { adenosepalum Murb. }\end{array}$ & Scrophulariaceae & Sığırkuyruğu & Leaf & Dec., Int. & [6] \\
\hline Verbascum sp. & Scrophulariaceae & $\begin{array}{l}\text { Siğırkuyruğu, } \\
\text { Tozluk, } \\
\text { Tozkulak, Calba }\end{array}$ & $\begin{array}{l}\text { Aerial part } \\
\text { Flower }\end{array}$ & $\begin{array}{l}\text { +Tavşan otu } \\
\text { (Heracleum } \\
\text { platytaenium } \\
\text { Boiss.) aerial part, } \\
\text { Inf., Enema } \\
\text { Inf. and Mush Int. } \\
\text { Dec., Sediment } \\
\text { Ext. }\end{array}$ & {$[33]$} \\
\hline V. speciosum Schrader & Scrophulariaceae & $\begin{array}{l}\text { Kabalak, } \\
\text { Ayılahanası }\end{array}$ & $\begin{array}{l}\text { Flower } \\
\text { Root }\end{array}$ & $\begin{array}{l}\text { Inf., Int. } \\
\text { Like a pill } \\
\text { Swallowed }\end{array}$ & [47] \\
\hline V. thapsus L. & Scrophulariaceae & Sığırkuyruğu & $\begin{array}{l}\text { Branch, Stem, } \\
\text { Flower }\end{array}$ & Inf., Int. & [29] \\
\hline Verbena officinalis L. & Verbenaceae & $\begin{array}{l}\text { Basur otu, Basir } \\
\text { otu }\end{array}$ & Aerial part & $\begin{array}{l}\text { Dried, Crushed, } \\
\text { Eaten } \\
\text { Dec., Int. } \\
\text { Raw, Eaten } \\
\text { Ext. }\end{array}$ & {$[71]$} \\
\hline Viscum album L. & Loranthaceae & $\begin{array}{l}\text { Ökse otu, } \\
\text { Gövelek, } \\
\text { Gövem }\end{array}$ & $\begin{array}{l}\text { Whole plant } \\
\text { Leafy branch }\end{array}$ & $\begin{array}{l}\text { Dec., Int. } \\
\text { Dec. and Inf., } \\
\text { Crushed, +Water, } \\
\text { Mac. }\end{array}$ & $\begin{array}{l}{[11]} \\
{[53]}\end{array}$ \\
\hline V. album L. subsp. album & Loranthaceae & $\begin{array}{l}\text { Ökse otu, } \\
\text { Yapışkan ot, } \\
\text { Çeküm }\end{array}$ & $\begin{array}{l}\text { Whole plant } \\
\text { Leaf }\end{array}$ & $\begin{array}{l}\text { Dec., Int. } \\
\text { Inf., Int. } \\
\text { Dried, Inf., Int. } \\
\text { Dec., Ext. }\end{array}$ & $\begin{array}{l}{[6]} \\
{[64]} \\
{[81]} \\
{[19]}\end{array}$ \\
\hline Vitex agnus-castus L. & Verbenaceae & Hayit & Seed & Dec., Int. & {$[58]$} \\
\hline Vitis sylvestris L. & Vitaceae & Asma, Üzüm & Fruit, Leaf & $\begin{array}{l}\text { Raw and Dec., } \\
\text { Int. and Ext. }\end{array}$ & {$[17]$} \\
\hline$V$. vinifera L. & Vitaceae & Üzüm & Fruit & Swallowed & {$[64]$} \\
\hline Xanthium strumarium L. & Asteraceae & Domuz pitrağı & Fruit & Mush, Int. & [33] \\
\hline Zea mays L. & Poaceae & Misır, Lazut & Stylus & $\begin{array}{l}\text { Dec., Int. } \\
\text { Inf., Int. }\end{array}$ & $\begin{array}{l}{[35]} \\
{[86]}\end{array}$ \\
\hline
\end{tabular}

Int.: Internal, Ext.: External, Dec.: Decoction, Inf.: Infusion, Mac.: Maceration 


\section{MATERIALS AND METHODS}

This study has been prepared by searching thesis at the National Higher Education Center and ethnobotanical surveys conducted in various parts of Turkey with selecting regional plants used for hemorrhoids treatment. Our study includes ethnobotanical surveys made after 2004. The surveys prepared before 2004 were reported by Gürhan G. and Ezer N. [3].

\section{RESULTS}

This study, prepared by screening of ethnobotanical researches, revealed 241 taxa belonging 62 families that were used against hemorrhoids among the population. These plants are mainly from Asteraceae (40 taxa), Lamiaceae (23 taxa), Rosaceae (19 taxa), Scrophulariaceae (12 taxa), Araceae (8 taxa), Polygonaceae (8 taxa) and Cupressaceae (7 taxa) (Figure 1). The most common species in ethnobotanical studies in different parts of Turkey are Achillea sp., Arum sp., Cichorium intybus L., Dracunculus vulgaris Schott, Ecballium elaterium (L.) A. Rich., Ficus carica L., Hypericum perforatum L., H. scabrum L., Juglans regia L., Peganum harmala L., Rosa canina L., Rubus sp., Sambucus ebulus L., S. nigra L., Teucrium polium L., Urtica dioica L., Verbascum sp. Our study includes 178 different taxa than previously reported [3].

Plant parts such as leaves, flowers containing volatile oil are prepared as infusion; seeds, roots are prepared as decoction; or they are prepared in form a pill. These forms are used internally. It is used externally by preparing mush, sitting in its vapour or preparing a bath.

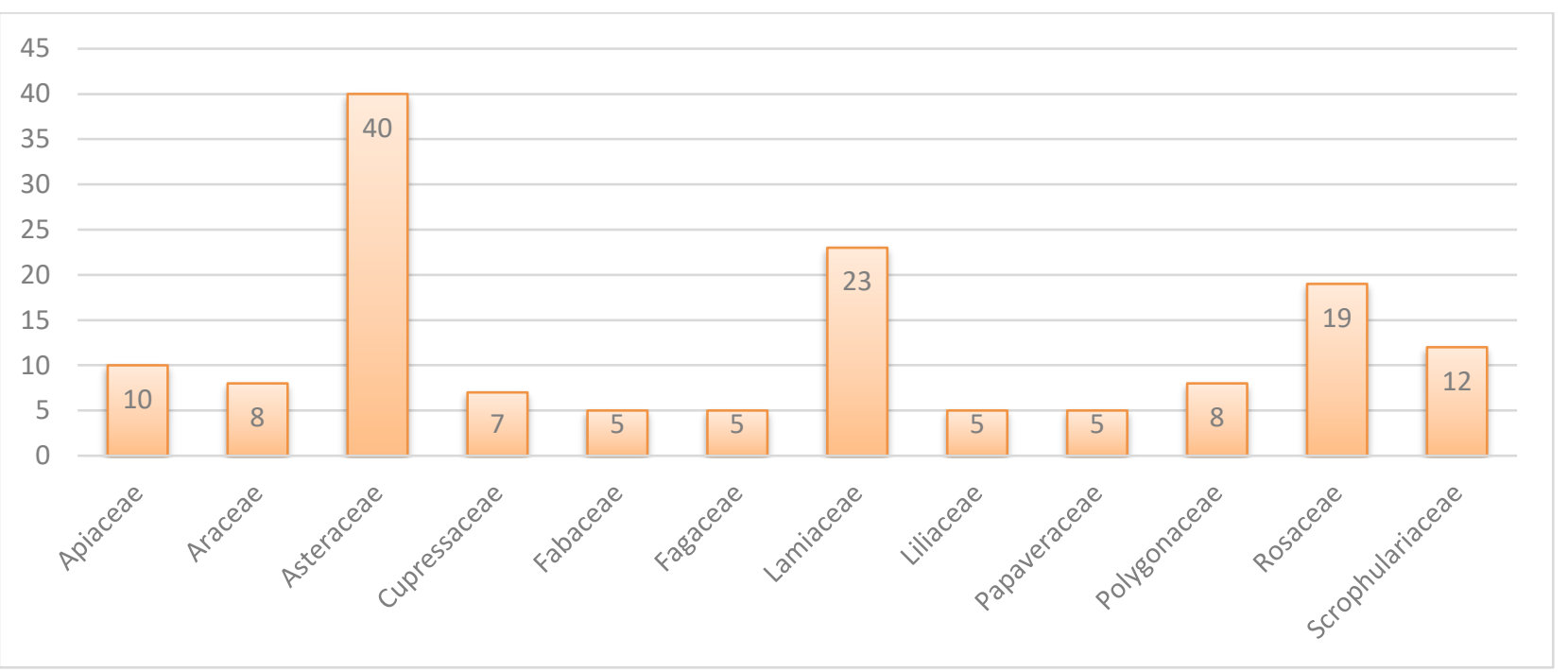

Figure 1. Main families used in traditional treatment against hemorrhoids in Turkey.

It is estimated that plants are used in hemorrhoids treatment because of their analgesic, antiinflammatory, antimicrobial, antiseptic, wound healing and vitamin P effects. Plants rich with flavonoid compounds (eg. Sambucus sp., Rosa canina, Rubus sp.) have vitamin P activity. Due to vitamin P effect, flavonoids strengthen the vessel walls, reduce capillary vascular permeability and prevent bleeding. Owing to this effect, capillary hemorrhages seen in hemorrhoids can be reduced. It is thought that hemorrhoid complaints can be healed with wound healing compounds (including plants eg. Achillea sp., Arum sp., Dracunculus vulgaris, Ecballium elaterium, Ficus carica, Hypericum sp.); analgesic, antiinflammatory compounds (including plants eg. Salix alba, Cichorium intybus); antimicrobial, antiseptic compounds (including plants eg. Peganum harmala, Teucrium polium, Urtica dioica, Verbascum sp.) [97; 98; 99; 100]. More detailed studies on the chemical components and biological activities of plants used in hemorrhoids treatment can be made. We hope that our study will contribute on the development of new drugs to be used in the treatment of hemorrhoids.

Author contributions: Concept - M.Ş.E., A.S.; Design - M.Ş.E., A.S.; Supervision - M.Ş.E., A.S.; Resource - M.Ş.E., A.S.; Materials - M.Ş.E., A.S.; Data Collection and/or Processing - M.Ş.E., A.S.; Analysis and/or Interpretation - M.Ş.E., A.S.; Literature Search - M.Ş.E., A.S.; Writing - M.Ş.E., A.S.; Critical Reviews - M.Ş.E., A.S.

Conflict of interest statement: The authors declared no conflict of interest. 


\section{REFERENCES}

[1] Kahn A and Jewell T. What are hemorrhoids? Available in: http://www.healthline.com/health/hemorrhoids?m=0 [Accessed: 24.03.2017].

[2] Grup Florence Nightingale Hastaneleri. Hemoroit tedavisi. Available in: https://www.florence.com.tr/hastaliktedavisi/detay/hemoroid-tedavisi [Accessed: 24.03.2017].

[3] Gürhan G, Ezer N. Halk arasında hemoroit tedavisinde kullanılan bitkiler-I. Hacettepe Üni Ecz Fak Derg. 2004; 24(1): 37-55.

[4] Özdemir E, Alpınar K. An ethnobotanical survey of medicinal plants in western part of Central Taurus Mountains: Aladaglar (Nigde - Turkey). J Ethnopharmacol. 2015; 166: 53-65.

[5] Aktan T. MSc Thesis. The Ethnobotanical examine in the villages of Yenişehir (Bursa). Department of Biology, Celal Bayar University, Manisa, 2011.

[6] Doğan A. PhD Thesis. Pertek (Tunceli) yöresinde etnobotanik araştırmalar. Department of Pharmaceutical Botany, Marmara University, İstanbul, 2014.

[7] Güneş S. MSc Thesis. Karaisalı (Adana) and villages natural plants used by the public, ethnobotanical respect investigation. Department of Biology, Niğde University, Niğde, 2010.

[8] Koca C. MSc Thesis. Aktarların tüketici profillerinin ve satın alma eğilimlerinin belirlenmesi (Kahramanmaraş örneği). Department of Forest Engineering, Sütçü İmam University, Kahramanmaraş, 2016.

[9] Akbulut S, Bayramoğlu MM. The trade and use of medical and aromatic herbs in Turkey. Ethno Med. 2013; 7(2): 6777.

[10] Polat R, Cakilcioglu U, Kaltalioglu K, Ulusan MD, Türkmen Z. An ethnobotanical study on medicinal plants in Esipiye and its surrounding (Giresun - Turkey). J Ethnopharmacol. 2015; 163: 1-11.

[11] Saraç DU. MSc Thesis. Ethnobotanic features of Rize province. Forest Engineering Graduate Program, Karadeniz Technical University, Trabzon, 2013.

[12] Saraç DU, Özkan ZC, Akbulut S. Ethnobotanic features of Rize/Turkey province. Biol Divers Conserv 2013; 6(3): 5766.

[13] Kültür Ş. Medicinal plants used in Kırklareli province (Turkey). J Ethnopharmacol. 2007; 111: 341-364.

[14] Emre Bulut G. PhD Thesis. Ethnobotanical investigations in Bayramiç (Çanakkale). Department of Pharmaceutical Botany, Marmara University, İstanbul, 2008.

[15] Deniz L. PhD Thesis. The flora and its ethnobotanic evaluation of Usak University 1 Eylül Campus (Usak). Department of Biology, Afyon Kocatepe University, Afyon, 2008.

[16] Deniz L, Serteser A, Kargığlu M. Uşak Üniversitesi ve yakın çevresindeki bazı bitkilerin mahalli adları ve etnobotanik özellikleri. Afyon Kocatepe Üni Fen Bil Derg. 2010; 1: 57-72.

[17] Kilic O, Bagci E. An ethnobotanical survey of some medicinal plants in Keban (Elazı̆ - Turkey). J Med Plants Res. 2013; 7(23): 1675-1684.

[18] Cakilcioglu U, Khatun S, Turkoglu I, Hayta S. Ethnopharmacological survey of medicinal plants in Maden (Elazig Turkey). J Ethnopharmacol. 2011; 137: 469-486.

[19] Altundag E, Ozturk M. Ethnomedicinal studies on the plant resources of East Anatolia, Turkey. Procedia Soc Behav Sci. 2011; 19: 756-777.

[20] Güzelşemme M. MSc Thesis. Antakya'da kullanılan tıbbi bitkiler ile yabani gıda bitkileri, Department of Biology, Mustafa Kemal University, Hatay, 2014.

[21] Güzel Y, Güzelşemme M, Miski M. Ethnobotany of medicinal plants used in Antakya: A multicultural district in Hatay province of Turkey. J Ethnopharmacol. 2015; 174: 118-152.

[22] Altundağ E. PhD Thesis. Iğdır ilinin (Doğu Anadolu Bölgesi) doğal bitkilerinin halk tarafından kullanımı. Department of Pharmaceutical Botany, Istanbul University, İstanbul, 2009.

[23] Öztürk M. MSc Thesis. The flora and ethnobotany of Nizip region (Aksaray). Department of Biology, Selçuk University, Konya, 2006.

[24] Demirci S. MSc Thesis. Ethnobotanical study in Andırın (Kahramanmaraş) district. Department of Pharmaceutical Botany, İstanbul University, Istanbul, 2010.

[25] Mart S. MSc Thesis. An ethnobotanical investigation of the natural plants using by inhabitants in Bahçe and Hasanbeyli districts of Osmaniye province. Department of Biology, Çukurova University, Adana, 2006.

[26] Güneş F, Özhatay N. An ethnobotanical study from Kars (Eastern) Turkey. Biol Divers Conserv. 2011; 4(1): 30-41.

[27] Arı S. PhD Thesis. Afyonkarahisar ve civarında halk tarafından kullanılan bazı bitkilerin etnobotanik özellikleri. Department of Moleculer Biology and Genetic, Afyon Kocatepe University, Afyon, 2014. 
[28] Karcı E. MSc Thesis. Bafra (Samsun) halk ilaçları. Department of Pharmacognosy, Gazi University, Ankara, 2013.

[29] Karakurt E. MSc Thesis. Kelkit (Gümüşhane) ilçesinin etnobotanik özellikleri. Department of Biology, Erzincan University, Erzincan, 2014.

[30] Şenkardeş İ. PhD Thesis. Ethnobotanical investigations in southern districts (Acıgöl, Derinkuyu, Gülşehir, NevşehirCentral district, Ürgüp) of Nevşehir. Department of Pharmaceutical Botany, Marmara University, Istanbul, 2014.

[31] Polat R, Cakılcioglu U, Satıl F. Traditional uses of medicinal plants in Solhan (Bingöl-Turkey). J Ethnopharmacol. 2013;148: 951-963.

[32] Ecevit Genç G. MSc Thesis. Çatalca yöresinde etnobotanik bir araştırma. Department of Pharmaceutical Botany, Istanbul University, Istanbul, 2003.

[33] Sargın SA, Akçiçek E, Selvi S. An ethnobotanical study of medicinal plants used by the local people of Alaşehir (Manisa) in Turkey. J Ethnopharmacol. 2013; 150(3): 860-874.

[34] Uzun Yılmaz Y. MSc Thesis. Beşikdüzü yöresinde gıda amaçlı kullanılan bitkiler. Department of Forest Engeneering, Karadeniz Technical University, Trabzon, 2011.

[35] Koyuncu O. PhD Thesis. Investigations of floristical and etnobotanical aspect of Geyve (Sakarya) and its environs. Department of Biology, Eskişehir Orhangazi University, Eskişehir, 2005.

[36] Gürdal B. MSc Thesis. Ethnobotanical study in Marmaris district (Muğla). Department of Pharmaceutical Botany, Istanbul University, İstanbul, 2010.

[37] Gürdal B, Kültür Ş. An ethnobotanical study of medicinal plants in Marmaris (Muğla, Turkey). J Ethnopharmacol. 2013; 146: 113-126.

[38] Mükemre M. MSc Thesis. Ethnobotanical features of Konalga, Sırmalı, Dokuzdam villages (Çatak/ Van) and their vicinity. Department of Biology, Yüzüncü Y1l University, Van, 2013.

[39] Mükemre M, Behçet L, Çakılcığlu U. Ethnobotanical study on medicinal plants in villages of Çatak (Van - Turkey). J Ethnopharmacol. 2015; 166: 361-374.

[40] Kaval İ. MSc Thesis. Ethnobotanical features of Geçitli (Hakkari) and surroundings. Department of Biology, Yüzüncü yıl University, Van, 2011.

[41] Kaval İ, Behçet L, Cakilcioglu U. Ethnobotanical study on medicinal plants in Geçitli and its surrounding (Hakkari Turkey). J Ethnopharmacol. 2014; 155(1): 171-184.

[42] Sargin SA. PhD Thesis. Agricultural biodiversity and ethnobotanical survey of Alaşehir (Manisa) and its surrounding area. Department of Biology, Balıkesir University, Balıkesir, 2013.

[43] Akgül A. MSc Thesis. Ethnobotany at Midyat (Mardin). Department of Biology, Ege University, İzmir, 2008.

[44] Metin A. Msc Thesis. Ethnobotanical features of plants in Mut (Mersin)and its environments. Department of Biology, Selçuk University, Konya, 2009.

[45] Erdoğan R. MSc Thesis. Ethnobotanical features some of the wild on the Sarıveliler (Karaman) and its environment ethnobotanic. Department of Biology, Selçuk University, Konya, 2011.

[46] Bağcı Y, Erdoğan R, Doğu S. Sarıveliler (Karaman) ve çevresinde yetişen bitkilerin etnobotanik özellikleri. Selçuk Üni Fen Fak Derg. 2016; 42(1): 84-107.

[47] Kızılarslan Ç. MSc Thesis. An ethnobotanical survey in the South part of İzmit Gulf. Department of Pharmaceutical Botany, İstanbul University, İstanbul, 2008.

[48] Kızılarslan Ç, Özhatay N. Wild plants used as medicinal purpose in the south part of İzmit (Northwest Turkey). Turk J Pharm Sci. 2012; 9(2): 199-218.

[49] Fakir H, Korkmaz M, Güller B. Medicinal plant diversity of Western Mediterrenean region in Turkey. J Appl Biol Sci. 2009; 3(2): 30-40.

[50] Demirci S, Özhatay N. An ethnobotanical study in Kahramanmaraş (Turkey); Wild plants used for medicinal purpose in Andırın, Kahramanmaraş. Turk J Pharm Sci. 2012; 9(1): 75-92.

[51] Saday H. MSc Thesis. Ethnobotanical properties of Güzeloluk village and its vicinity. Department of Biology, Selçuk University, Konya, 2009.

[52] Eşen B. MSc Thesis. The ethnobotanical properties of Aydınlar village and its vicinity (Erdemli/ Mersin). Department of Biology, Selçuk University, Konya, 2008.

[53] Oral ÇD. Msc Thesis. Ethnobotanical studies on folk medicines used in Konya. Program Phytotherapy, Gazi University, Ankara, 2007.

[54] Özüdoğru B, Akaydın G, Erik S, Yeşilada E. Inferences from an ethnobotanical field expedition in the selected locations of Sivas and Yozgat provinces (Turkey). J Ethnopharmacol. 2011; 137: 85-98.

[55] Yeşil Y, Akalın E. Folk medicinal plants in Kürecik area (Akçadağ/Malatya - Turkey). Turkish J Pharm Sci. 2009; 3 : 207-220. 
[56] Sağıroğlu M, Arslantürk A, Akdemir ZK, Turna M. An ethnobotanical from Hayrat (Trabzon) and Kalkandere (Rize/Turkey). Biol Divers Conserv. 2012; 5(1): 31-43.

[57] Güler B, Manav E, Uğurlu E. Medicinal plants used by traditional healers in Bozüyük (Bilecik - Turkey). J Ethnopharmacol. 2015; 173: 39-47.

[58] Vural G. MSc Thesis. Ethnobotanical features some of the willd plants on the Honaz mountain and its environment ethnobotanic. Department of Biology, Afyon Kocatepe University, Afyon, 2008.

[59] Mumcu Arısan Ö. PhD Thesis. Işık Dağı ve çevresinde yetişen bitkiler üzerinde farmasötik botanik yönünden araştırmalar. Department of Pharmaceutical Botany, Ankara University, Ankara, 2010.

[60] Tetik F. MSc Thesis. A research on the ethnobotanical valued plants in Malatya province. Firat University, Elazı $\breve{g}$, 2011.

[61] Tetik F, Civelek S, Cakilcioglu U. Traditional uses of some medicinal plants in Malatya (Turkey). J Ethnopharmacol. 2013; 146: 331-346.

[62] Polat R, Satıl F. An ethnobotanical survey of medicinal plants in Edremit Gulf (Balıkesir - Turkey). J Ethnopharmacol. 2012; 139: 626-641.

[63] Han Mí. MSc Thesis. Kadışehri (Yozgat) yöresinin geleneksel halk ilacı olarak kullanılan bitkileri. Department of Pharmaceutical Botany, Marmara University, Istanbul, 2012.

[64] Bulut G, Tuzlacı E. An ethnobotanical study of medicinal plants in Turgutlu (Manisa - Turkey). J Ethnopharmacol. 2013; 149: 633-647.

[65] Polat R. PhD Thesis. Agricultural biodiversity and ethnobotanical research in the Havran and Burhaniye regions of Balıkesir. Department of Biology, Balıkesir University, Balıkesir, 2010.

[66] Alkaç SA. MSc Thesis. Alaçam mountains (Balıkesir) from Bigadic city area around economic importance of some plants and features ethnobotany. Department of Biology, Balıkesir University, Balıkesir, 2013.

[67] Akyol Y, Altan Y. Ethnobotanical studies in the Maldan Village (Province Manisa, Turkey). Marmara Pharm J. 2013; 17: 21-25.

[68] Ugurlu E, Secmen O. Medicinal plants popularly used in villages of Yunt Mountain (Manisa- Turkey). Fitoterapia 2008; 79: 126-131.

[69] Onar S. MSc Thesis, Bandırma (A1(A), Balıkesir) ve çevresinin etnobotaniği. Department of Biology, Çanakkale Onsekiz Mart University, Çanakkale, 2006.

[70] Sarper F, Akaydın G, Şimşek I, Yeşilada E. An ethnobotanical field survey in the Haymana district of Ankara Province in Turkey. Turk J Biol. 2009; 33: 79-88.

[71] Keskin L. MSc Thesis. Kadınhanı (Konya) ve çevresinde yetişen bitkilerin etnobotanik özellikleri. Department of Biology, Selçuk University, Konya, 2011.

[72] Behçet L, Arık M. An ethnobotanical investigation in East Anatolia (Turkey). Türk Doğa Fen Derg. 2013; 2(1): 1-14.

[73] Şenkardeş İ, Tuzlacı E. Some ethnobotanical notes from Gündoğmuş district (Antalya/Turkey). MÜSBED. 2014; 4(2): 63-75.

[74] Kökçü B. MSc Thesis. Lapseki (A1/A), Çanakkale, Türkiye) ve çevresinin etnobotaniği. Department Biology, Çanakkale Onsekiz Mart Univeristy, Çanakkale, 2015.

[75] Koçyiğit M. MSc Thesis. Yalova ilinde etnobotanik bir araştırma. Department of Pharmaceutical Botany, Istanbul University, Istanbul, 2005.

[76] Korkmaz M, Karakurt E. Kelkit (Gümüşhane) aktarlarında satılan tıbbi bitkiler. Süleymen Demirel Üni Fen Bil Enst Derg. 2014; 18(3): 60-80.

[77] Sargin SA, Selvi S, Lopez V. Ethnomedicinal plants of Sarigöl district (Manisa), Turkey. J Ethnopharmacol. 2015; 171: 64-84.

[78] Özkan AM. PhD Thesis. Pınarbaşı (Kayseri) florası üzerinde farmasötik botanik yönünden araştırmalar, Department of Pharmaceutical Botany, Ankara University, Ankara, 2002.

[79] Hayta S, Polat R, Selvi S. Traditional uses of medicinal plants in Elazı̆̆ (Turkey). J Ethnopharmacol. 2014; 154: 613623.

[80] Dağlı M. MSc Thesis. Şanlıurfa merkez ve bağlı köylerde etnobotanik bir araştırma. Department of Biology, Harran University, Şanlıurfa, 2015.

[81] Kurnaz Karagöz F. MSc Thesis. Suşehri (Sivas) bölgesinin etnobotanik açıdan değerlendirilmesi. Department of Biology, Afyon Kocatepe University, Afyon, 2013.

[82] Bulut Y. MSc Thesis. Manavgat (Antalya) yöresinin faydalı bitkileri. Departmant of Biology, Süleyman Demirel University, Isparta, 2006. 
[83] Şenkardeş İ. MSc Thesis. Ürgüp (Nevşehir) yöresinin geleneksel halk ilacı olarak kullanılan bitkileri. Department of Pharmaceutical Botany, Marmara University, Istanbul, 2010.

[84] Uysal G. MSc Thesis. Köyceğiz (Muğla) ilçesinin etnobotaniği. Department of Biology, Muğla University, 2008.

[85] Balos MM. MSc Thesis. The flora and ethnobotany of the region between Zeytinbahçe and Akarçay (Birecik). Department of Biology, Harran University, Şanlıurfa, 2007.

[86] Özgökçe F, Özçelik H. Ethnobotanical aspects of some taxa in East Anatolia, Turkey. Econ Bot 2004; 58 (7): $697-704$.

[87] Yüzbaşıŏlu E. MSc Thesis. Reşadiye (A6, Tokat, Türkiye) ve çevresinin etnobotaniği. Department of Biology, Çanakkale Onsekiz Mart University, Çanakkale, 2010.

[88] Ayan Ö. MSc Thesis. Antimicrobial activities of some edible plant taxa which are used as ethnobotonical purpose in Kastamonu region, on some food pathogens. Department of Forest Engineering, Kastamonu University, Kastamonu, 2015.

[89] Bakır Sade Y. MSc Thesis. Kahta (Adıyaman) merkezi ve Narince köyünün etnobotanik açıdan araştırılması. Department of Biology, Harran University, Şanlıurfa, 2014.

[90] Alpaslan Z. MSc Thesis. The ethnobotanical properties of Ergan mountain (Erzincan). Department of Biology, Erzincan University, Erzincan, 2012.

[91] Tekin S. MSc Thesis. Ethnobotanic Aspects of Üzümlü (Erzincan) Town. Department of Biology, Erzincan University, Erzincan, 2011.

[92] Kazan D. MSc Thesis. Ortaca (Muğla) ilçesinin etnobotaniği. Department of Biology, Muğla University, 2007.

[93] Kural K. MSc Thesis. Trabzon çevresinde yayılış gösteren faydalı bitkiler üzerinde ekonomik botanik yönünden araştırmalar. Department of Biology, Istanbul University, Istanbul, 2012.

[94] Koçyiğit M, Özhatay N. Wild plants used as medicinal purpose in Yalova (Northwest Turkey). Turkish J Pharm Sci. 2006; 3(2): 91-103.

[95] Akan H, Bakır Sade Y. Kahta (Adıyaman) merkez ve Narince Köyü'nün etnobotanik açıdan araştırılması. BEÜ Fen Bil Derg. 2015; 4(2): 219-248.

[96] Günbatan T. MSc Thesis. Çamlıdere (Ankara) halk ilaçları. Department of Pharmacognosy, Gazi University, Ankara, 2011.

[97] Baytop T, Türkiye'de Bitkiler ile Tedavi, Geçmişte ve Bugün, Nobel Medicine Publication, Istanbul 1999.

[98] Street RA, Sidana J, Prinsloo G. Cichorium inthybus: Traditional uses, phytochemistry, pharmacology and toxicology. J Evid Based Complementary Altern Med. 2013; Article ID 579319.

[99] Bahramikia S, Yazdanparast R. Phytochemistry and medicinal properties of Teucrium polium L. (Lamiaceae). Phytother Res. 2012; 26: 1581-1593.

[100] Süntar I, Tatlı I, Küpeli Akkol E, Keleş H, Kahraman Ç, Akdemir Z. An ethnopharmacological study on Verbascum species: From conventional wound healing use to scientific verification. J Ethnopharmacol. 2010; 132: 408-413. 